\title{
The maximal degree of the Khovanov homology of a cable link
}

\author{
KEIJI TAGAMI
}

In this paper, we study the Khovanov homology of cable links. We first estimate the maximal homological degree term of the Khovanov homology of the $(2 k+1,(2 k+1) n)$-torus link and give a lower bound of its homological thickness. Specifically, we show that the homological thickness of the $(2 k+1,(2 k+1) n)$-torus link is greater than or equal to $k^{2} n+2$. Next, we study the maximal homological degree of the Khovanov homology of the $(p, p n)$-cabling of any knot with sufficiently large $n$. Furthermore, we compute the maximal homological degree term of the Khovanov homology of such a link with even $p$. As an application we compute the Khovanov homology and the Rasmussen invariant of a twisted Whitehead double of any knot with sufficiently many twists.

57M27; 57M25

\section{Introduction}

A knot is an embedding of a circle into the 3-sphere. A link is an embedding of a disjoint union of finitely many circles into the 3 -sphere.

In [6], for each link $L$, Khovanov defined a graded chain complex whose graded Euler characteristic is equal to the Jones polynomial of $L$. Its homology group is a link invariant and called the Khovanov homology. Khovanov homology has two gradings, homological degree $i$ and $q$-grading $j$. In this paper, we denote the homological degree- $i$ term of the Khovanov homology of $L$ by $\mathrm{KH}^{i}(L)$ and denote the homological degree- $i$ and $q$-grading $j$ term of the Khovanov homology of $L$ by $\mathrm{KH}^{i, j}(L)$.

The $(p, q)$-cabling $K(p, q)$ of a knot $K$ is the satellite link with companion $K$ and pattern the $(p, q)$-torus link $T_{p, q}$. The Alexander polynomial of a cable link satisfies the following formula (see Lickorish [10]):

$$
\Delta_{K(p, q)}(t)=\Delta_{K}\left(t^{p}\right) \Delta_{T_{p, q}}(t) .
$$

The Jones polynomial of a cabling of $K$ is expressed in terms of the colored Jones polynomial of $K$. Indeed, the colored Jones polynomial has a cabling formula (for 
example, see Kirby and Melvin [8]). However, there are few works about the Khovanov homology (which is a categorification of the Jones polynomial) of cable links. The ( $2 k, 2 k n)$-torus link $T_{2 k, 2 k n}$ can be regarded as the $(2 k, 2 k n)$-cabling of the unknot and Stošić [15] showed that the maximal homological degree of the Khovanov homology of $T_{2 k, 2 k n}$ is $2 k^{2} n$ (Theorem 3.2). Moreover, he computed the homological degree$2 k^{2} n$ term (see Theorem 3.3).

In this paper, we consider the $(p, p n)$-cabling of any knot. Our main results are Theorems 1.1 and 1.3 below.

We first determine the maximal homological degree of the Khovanov homology of the $(2 k+1,(2 k+1) n)$-torus link $T_{2 k+1,(2 k+1) n}$ by Stošić's method. In addition, we determine the dimension of the maximal homological term of the Khovanov homology of such a link.

Theorem 1.1 Let $k$ and $n$ be positive integers. Denote the $(2 k+1,(2 k+1) n)$-torus link by $T_{2 k+1,(2 k+1) n}$. Assume that its orientation is given by the closure of the braid $\left(\sigma_{1} \cdots \sigma_{2 k}\right)^{(2 k+1) n}$ with all crossings positive, where the $\sigma_{i}$ are the standard generators of the braid group $B_{2 k+1}$. Then, for $i>2 k(k+1) n$, we have

$$
\mathrm{KH}^{i}\left(T_{2 k+1,(2 k+1) n}\right)=0 \text {. }
$$

On the other hand,

$$
\operatorname{dim}_{\mathbb{Q}} \mathrm{KH}^{2 k(k+1) n}\left(T_{2 k+1,(2 k+1) n}\right)=\left(\begin{array}{c}
2 k+2 \\
k+1
\end{array}\right) .
$$

Moreover, for $i=0, \ldots, k+1$, we have

$$
\mathrm{KH}^{2 k(k+1) n, 6 k(k+1) n+1-2 i}\left(T_{2 k+1,(2 k+1) n}\right) \neq 0 \text {. }
$$

From Theorem 1.1, we obtain the following.

Corollary 1.2 Let $k$ and $n$ be positive integers. Then we have

$$
\max \left\{i \in \mathbb{Z} \mid \mathrm{KH}^{i}\left(T_{2 k+1,(2 k+1) n}\right) \neq 0\right\}=2 k(k+1) n .
$$

Moreover, we also obtain an estimation of the homological thickness of $T_{2 k+1,(2 k+1) n}$ (see Corollary 3.12).

Next we consider the $(p, p n)-$ cabling $K(p, p n)$ of any oriented knot $K$. Assume that each component of $K(p, p n)$ has an orientation induced by $K$, that is, each component of $K(p, p n)$ is homologous to $K$ in the tubular neighborhood of $K$. For such a link, we obtain an analog of Theorem 1.1. 
Theorem 1.3 Let $K$ be an oriented knot and $D$ be a diagram of $K$ with $l_{+}$positive crossings and $l_{-}$negative crossings. Put $l=l_{+}+l_{-}$and $f=l_{+}-l_{-}$. Then for $n \geq l$ and any positive integer $k$, we obtain the following:

$$
\max \left\{i \in \mathbb{Z} \mid \mathrm{KH}^{i}(K(2 k, 2 k(n+f))) \neq 0\right\}=2 k^{2}(n+f) .
$$

In addition, if $n>l$, we determine the dimension of the maximal homological degree term of the Khovanov homology of the link:

$$
\operatorname{dim}_{\mathbb{Q}} \mathrm{KH}^{2 k^{2}(n+f)}(K(2 k, 2 k(n+f)))=\left(\begin{array}{c}
2 k \\
n
\end{array}\right) .
$$

Moreover, for $n>l$ and $i=0, \ldots, k$, we have

$$
\mathrm{KH}^{2 k^{2}(n+f), 6 k^{2}(n+f)-2 i}(K(2 k, 2 k(n+f))) \neq 0 .
$$

Corollary 1.2 and the first claim of Theorem 1.3 imply a relation between the number of full twists and the maximal degree of the Khovanov homology.

We also estimate the maximal homological degree of the Khovanov homology of the $(2 k+1,(2 k+1) n)$-cabling of any knot $K$.

Proposition 1.4 Let $K$ be an oriented knot and $D$ be a diagram of $K$ with $l_{+}$positive crossings and $l_{-}$negative crossings. Put $l=l_{+}+l_{-}$and $f=l_{+}-l_{-}$. Then for $n \geq l$ and any positive integer $k$, we have the following:

$$
\begin{aligned}
2 k(k+1)(n+f) & \leq \max \left\{i \in \mathbb{Z} \mid \mathrm{KH}^{i}(K(2 k+1,(2 k+1)(n+f))) \neq 0\right\} \\
& \leq 2 k(k+1)(n+f)+l_{+} .
\end{aligned}
$$

As an application, we can give a computation of the Khovanov homology of a twisted Whitehead double of any knot with sufficiently many twists (Proposition 5.2), since a cable link is obtained from such a knot by smoothing at a crossing. Moreover we compute the Rasmussen invariant $s$ [13] of such a knot (Corollary 5.6).

The paper is organized as follows: In Section 2, we recall the definition of Khovanov homology and our main tools. In Sections 3 and 4, we prove Theorems 1.1 and 1.3, and Proposition 1.4. In Section 5, we present our results on Whitehead doubles. The appendix contains the proofs of several technical results. 


\section{Khovanov homology}

\subsection{The definition of Khovanov homology}

In this subsection, we recall the definition of the (rational) Khovanov homology. Let $L$ be an oriented link. Take a diagram $D$ of $L$ and an ordering of the crossings of $D$. For each crossing of $D$, we define a 0 -smoothing and a 1 -smoothing as in Figure 1. A smoothing of $D$ is a diagram where each crossing of $D$ is changed by either 0 -smoothing or 1 -smoothing.

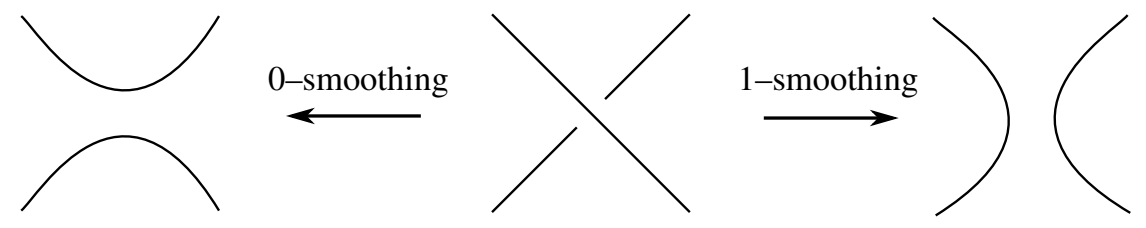

Figure 1: $0-$ smoothing and 1-smoothing

Let $n$ be the number of the crossings of $D$. Then $D$ has $2^{n}$ smoothings. By using the given ordering of the crossings of $D$, we have a natural bijection between the set of smoothings of $D$ and the set $\{0,1\}^{n}$, where, to any $\varepsilon=\left(\varepsilon_{1}, \ldots, \varepsilon_{n}\right) \in\{0,1\}^{n}$, we associate the smoothing $D_{\varepsilon}$, where the $i^{\text {th }}$ crossing of $D$ is $\varepsilon_{i}$-smoothed. Each smoothing $D_{\varepsilon}$ is a collection of disjoint circles.

Let $V$ be a graded free $\mathbb{Q}$-module generated by 1 and $X$ with $\operatorname{deg}(1)=1$ and $\operatorname{deg}(X)=-1$. Let $k_{\varepsilon}$ be the number of the circles of the smoothing $D_{\varepsilon}$. Put $M_{\varepsilon}=$ $V^{\otimes k_{\varepsilon}}$. The module $M_{\varepsilon}$ has a graded module structure, that is, for $v=v_{1} \otimes \cdots \otimes v_{k_{\varepsilon}}$ in $M_{\varepsilon}, \operatorname{deg}(v):=\operatorname{deg}\left(v_{1}\right)+\cdots+\operatorname{deg}\left(v_{k_{\varepsilon}}\right)$. Then define

$$
C^{i}(D):=\bigoplus_{|\varepsilon|=i} M_{\varepsilon}\{i\},
$$

where $|\varepsilon|=\sum_{i=1}^{m} \varepsilon_{i}$. Here, $M_{\varepsilon}\{i\}$ denotes $M_{\varepsilon}$ with its gradings shifted by $i$ (for a graded module $M=\bigoplus_{j \in \mathbb{Z}} M^{j}$ and an integer $i$, we define the graded module $M\{i\}=\bigoplus_{j \in \mathbb{Z}} M\{i\}^{j}$ by $\left.M\{i\}^{j}=M^{j-i}\right)$.

The differential map $d^{i}: C^{i}(D) \rightarrow C^{i+1}(D)$ is defined as follows. Fix an ordering of the circles for each smoothing $D_{\varepsilon}$ and associate the $i^{\text {th }}$ tensor factor of $M_{\varepsilon}$ to the $i^{\text {th }}$ circle of $D_{\varepsilon}$. Take elements $\varepsilon$ and $\varepsilon^{\prime} \in\{0,1\}^{n}$ such that $\varepsilon_{j}=0$ and $\varepsilon_{j}^{\prime}=1$ for some $j$ and that $\varepsilon_{i}=\varepsilon_{i}^{\prime}$ for any $i \neq j$. For such a pair $\left(\varepsilon, \varepsilon^{\prime}\right)$, we will define a map $d_{\varepsilon \rightarrow \varepsilon^{\prime}}: M_{\varepsilon} \rightarrow M_{\mathcal{E}^{\prime}}$. 
In the case where two circles of $D_{\varepsilon}$ merge into one circle of $D_{\varepsilon^{\prime}}$, the map $d_{\varepsilon \rightarrow \varepsilon^{\prime}}$ is the identity on all factors except the tensor factors corresponding to the merged circles, where it is a multiplication map $m: V \otimes V \rightarrow V$ given by:

$$
m(1 \otimes 1)=1, \quad m(1 \otimes X)=m(X \otimes 1)=X, \quad m(X \otimes X)=0 .
$$

In the case where one circle of $D_{\varepsilon}$ splits into two circles of $D_{\varepsilon^{\prime}}$, the map $d_{\varepsilon \rightarrow \varepsilon^{\prime}}$ is the identity on all factors except the tensor factor corresponding to the split circle where it is a comultiplication map $\Delta: V \rightarrow V \otimes V$ given by:

$$
\Delta(1)=1 \otimes X+X \otimes 1, \quad \Delta(X)=X \otimes X .
$$

If there exist distinct integers $i$ and $j$ such that $\varepsilon_{i} \neq \varepsilon_{i}^{\prime}$ and that $\varepsilon_{j} \neq \varepsilon_{j}^{\prime}$, then define $d_{\varepsilon \rightarrow \varepsilon^{\prime}}=0$.

In this setting, we define a map $d^{i}: C^{i}(D) \rightarrow C^{i+1}(D)$ by $\sum_{|\varepsilon|=i} d_{\varepsilon}^{i}$, where the map $d_{\varepsilon}^{i}: M_{\varepsilon} \rightarrow C^{i+1}(D)$ is defined by

$$
d^{i}(v):=\sum_{\left|\varepsilon^{\prime}\right|=i+1}(-1)^{l\left(\varepsilon, \varepsilon^{\prime}\right)} d_{\varepsilon \rightarrow \varepsilon^{\prime}}(v) .
$$

Here $v \in M_{\varepsilon} \subset C^{i}(D)$ and $l\left(\varepsilon, \varepsilon^{\prime}\right)$ is the number of 1 in front of (in our order) the factor of $\varepsilon$ which is different from $\varepsilon^{\prime}$.

We can check that $\left(C^{i}(D), d^{i}\right)$ is a cochain complex and we denote its $i^{\text {th }}$ homology group by $H^{i}(D)$. We call these the unnormalized homology groups of $D$. Since the map $d^{i}$ preserves the grading of $C^{i}(D)$, the group $H^{i}(D)$ has a graded structure $H^{i}(D)=\bigoplus_{j \in \mathbb{Z}} H^{i, j}(D)$ induced by that of $C^{i}(D)$. For any link diagram $D$, we define its Khovanov homology $\mathrm{KH}^{i, j}(D)$ by

$$
\mathrm{KH}^{i, j}(D)=H^{i+n_{-}, j-n_{+}+2 n_{-}}(D),
$$

where $n_{+}$and $n_{-}$are the number of the positive and negative crossings of $D$, respectively. The grading $i$ is called the homological degree and $j$ is called the $q$-grading.

Let $D$ and $D^{\prime}$ be link diagrams. The diagram $D$ is equivalent to $D^{\prime}$ if $D^{\prime}$ is obtained from $D$ by the Reidemeister moves (see Figure 2) and isotopies of the plane. It is known that two diagrams $D$ and $D^{\prime}$ are diagrams of the same link if and only if $D$ is equivalent to $D^{\prime}$. 


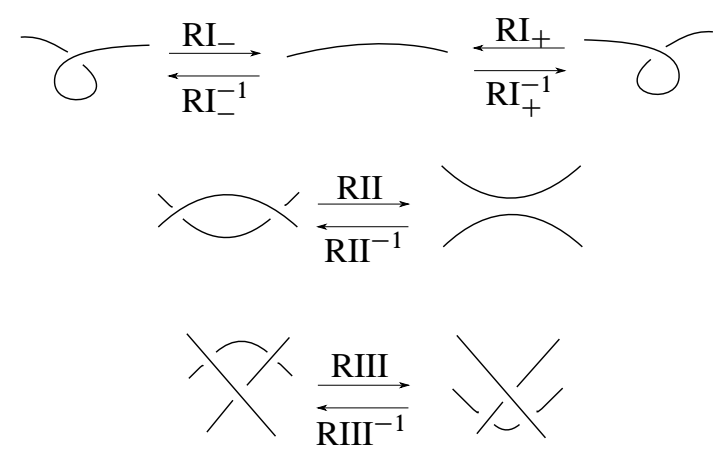

Figure 2: Reidemeister moves

Theorem 2.1 (Bar-Natan [3] and Khovanov [6]) Let $L$ be an oriented link and $D$ be a diagram of $L$. If $D^{\prime}$ is equivalent to $D$, the homology groups $\mathrm{KH}(D)$ and $\mathrm{KH}\left(D^{\prime}\right)$ are isomorphic. In this sense, we can denote $\mathrm{KH}(D)$ by $\mathrm{KH}(L)$. Moreover, the graded Euler characteristic of the homology $\mathrm{KH}(L)$ equals the Jones polynomial of $L$, that is,

$$
V_{L}(t)=\left.\left(q+q^{-1}\right)^{-1} \sum_{i, j \in \mathbb{Z}}(-1)^{i} q^{j} \operatorname{dim}_{\mathbb{Q}} \mathrm{KH}^{i, j}(L)\right|_{q=-t^{\frac{1}{2}}},
$$

where $V_{L}(t)$ is the Jones polynomial of $L$.

\subsection{Main tools}

Our main tools are the following (Theorems 2.2 and 2.3 and Proposition 2.4).

2.2.1 A long exact sequence Let $D$ be a link diagram and $D_{i}$ be a diagram obtained from $D$ by $i$-smoothing at a crossing of $D$ (see Figure 3 ). The following exact sequence was introduced by Viro [18] (see also his [17]).
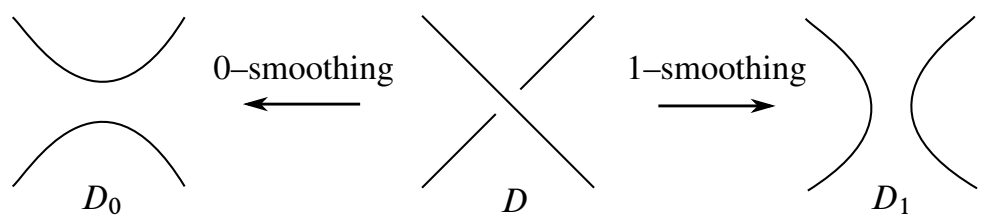

Figure 3: $D, D_{0}$ and $D_{1}$

Theorem 2.2 [18] There is a long exact sequence of the unnormalized homology groups:

$$
\cdots \longrightarrow H^{i-1, j-1}\left(D_{1}\right) \longrightarrow H^{i, j}(D) \longrightarrow H^{i, j}\left(D_{0}\right) \longrightarrow H^{i, j-1}\left(D_{1}\right) \longrightarrow \cdots .
$$


2.2.2 Lee homology Let $L$ be an oriented link. By Lee ${ }^{i}(L)$, we denote the homological degree- $i$ term of the Lee homology of $L$ (for details, see Lee [9]).

Theorem 2.3 [9] There is a spectral sequence whose $E_{\infty}$-page is the Lee homology and $E_{2}$-page is the Khovanov homology.

Proposition 2.4 [9, Proposition 4.3] Let $L$ be an oriented link with $n$ components, $S_{1}, \ldots, S_{n}$. Then we have

$$
\operatorname{dim}_{\mathbb{Q}}\left(\operatorname{Lee}^{i}(L)\right)=2\left\|\left\{E \subset\{2, \ldots, n\} \mid \sum_{j \in E, k \notin E} 2 \operatorname{lk}\left(S_{j}, S_{k}\right)=i\right\}\right\|,
$$

where $\operatorname{lk}\left(S_{j}, S_{k}\right)$ is the linking number of $S_{j}$ and $S_{k}$.

\section{The maximal degree of the Khovanov homology of the $(2 k+1,(2 k+1) n)$-torus link}

In this section, we prove Theorem 1.1, which has three claims. The first, second and third claims are Lemmas 3.7, 3.8 and 3.11 below, respectively. We first introduce some results by Stošić.

Definition 3.1 We denote the $(p, q)$-torus link by $T_{p, q}$. Put $D_{p, q}=\left(\sigma_{1} \cdots \sigma_{p-1}\right)^{q}$, where the $\sigma_{i}$ are the standard generators of the braid group $B_{p}$. The closure of the braid $D_{p, q}$ is a diagram of the $(p, q)$-torus link $T_{p, q}$. We give $T_{p, q}$ the downward orientation so that all crossings of $D_{p, q}$ are positive.

Stošić [15] showed the following results (Theorems 3.2 and 3.3 and Corollaries 3.4 and 3.5).

Theorem 3.2 [15, Theorem 1] Let $k$ and $n$ be positive integers. Then we have $\mathrm{KH}^{i}\left(T_{2 k, 2 k n}\right)=0$ if $i>2 k^{2} n$.

Theorem 3.3 [15, Theorem 3] Let $k$ and $n$ be positive integers. Then we have

$$
\operatorname{dim}_{\mathbb{Q}} \mathrm{KH}^{2 k^{2} n}\left(T_{2 k, 2 k n}\right)=\left(\begin{array}{c}
2 k \\
k
\end{array}\right) .
$$

Moreover, we obtain

$$
\operatorname{dim}_{\mathbb{Q}} \mathrm{KH}^{2 k^{2} n, 6 k^{2} n-2 i}\left(T_{2 k, 2 k n}\right)= \begin{cases}\left(\begin{array}{c}
2 k \\
k-i
\end{array}\right)-\left(\begin{array}{c}
2 k \\
k-i-1
\end{array}\right) & \text { if } i=0, \ldots, k \\
0 & \text { otherwise }\end{cases}
$$


From the above results, we can determine the maximal homological degree of the Khovanov homology of the $(2 k, 2 k n)$-torus link.

Corollary 3.4 [15] Let $k$ and $n$ be positive integers. Then we obtain

$$
\max \left\{i \in \mathbb{Z} \mid \mathrm{KH}^{i}\left(T_{2 k, 2 k n}\right) \neq 0\right\}=2 k^{2} n .
$$

Moreover we can estimate the homological thickness of the $(2 k, 2 k n)$-torus link.

Corollary 3.5 [15, Corollary 5] The homological thickness $\mathrm{hw}\left(T_{2 k, 2 k n}\right)$ of the $(2 k, 2 k n)$-torus link is greater than or equal to $k(k-1) n+2$, where the homological thickness hw $(L)$ of a link $L$ is defined as

$$
\frac{1}{2}\left(\max \left\{j-2 i \mid \mathrm{KH}^{i, j}(L) \neq 0\right\}-\min \left\{j-2 i \mid \mathrm{KH}^{i, j}(L) \neq 0\right\}\right)+1 .
$$

The homological thickness of a link estimates a distance between the link and an alternating link as follows. A link is $k$-almost alternating if it has a reduced diagram which can be alternating after $k$ crossing changes and no diagram which can be alternating after $k-1$ or less crossing changes (see [2]). Then we have the following results.

Theorem 3.6 [4, Theorem 8] Let $L$ be a $k$-almost alternating link. Then we obtain

$$
k \geq \operatorname{hw}(L)-2 .
$$

Remark From Corollary 3.5 and Theorem 3.6, the $(2 k, 2 k n)$-torus link has no diagram which is alternating after $k(k-1) n-1$ or less crossing changes.

Theorem 1.1 can be regarded as an analog of Theorems 3.2 and 3.3 and Corollary 3.4. Theorem 1.1 follows from Lemmas 3.7, 3.8 and 3.11 below. We will prove these lemmas.

Lemma 3.7 Let $k$ and $n$ be positive integers. Then we have $\mathrm{KH}^{i}\left(T_{2 k+1,(2 k+1) n}\right)=0$ if $i>2 k(k+1) n$.

Proof In Section 4, we prove Proposition 1.4, which implies Lemma 3.7.

Next we introduce Lemma 3.8. We can consider Lemma 3.8 to be an analog of the first claim of Theorem 3.3. 
Lemma 3.8 Let $k$ and $n$ be positive integers. Then we have

$$
\operatorname{dim}_{\mathbb{Q}} \mathrm{KH}^{2 k(k+1) n}\left(T_{2 k+1,(2 k+1) n}\right)=\left(\begin{array}{c}
2 k+2 \\
k+1
\end{array}\right) .
$$

To prove Lemma 3.8, we use the same notation as Stošić's in [14].

Definition 3.9 [14] Let $K$ be any positive braid link, that is, $K$ has a diagram which is the closure of a positive braid. Let $D$ be its diagram which is the closure of a positive braid with $p$ strands. The crossing $c$ of $D$ is of type $\sigma_{i}(i<p)$ if it corresponds to the generator $\sigma_{i}$ in the positive braid. Let $c_{1}^{i}, \ldots, c_{l_{i}}^{i}$ be the type- $\sigma_{i}$ crossings of $D$ and order them from top to bottom in the positive braid. Then we denote the crossing $c_{\alpha}^{i}$ by $(i, \alpha)$, where $1 \leq i \leq p$ and $1 \leq \alpha \leq l_{i}$.

Let $3 \leq p \leq q$. Let $E_{p, q}^{1}$ and $D_{p, q}^{1}$ be the diagrams obtained from $D_{p, q}$ by $1-$ smoothing and 0 -smoothing at the crossing $(p-1,1)$ of $D_{p, q}$, respectively. We continue the same process. Let $E_{p, q}^{2}$ and $D_{p, q}^{2}$ be the diagrams obtained from $D_{p, q}^{1}$ by 1 -smoothing and 0 -smoothing at the crossing $(p-2,1)$ of $D_{p, q}^{1}$ respectively. Repeating this process $p-1$ times, that is, for any $k=1, \ldots, p-1$, let $E_{p, q}^{k}$ and $D_{p, q}^{k}$ be the diagrams obtained from $D_{p, q}^{k-1}$ by 1 -smoothing and 0 -smoothing at the crossing $(p-k, 1)$ of $D_{p, q}^{k-1}$ respectively. Note that $D_{p, q}^{0}=D_{p, q}$ and that $D_{p, q}^{p-1}=D_{p, q-1}$. For example, see Figure 4.

We define $H^{i, j}\left(E_{p, q}^{k}\right):=H^{i, j}\left(\bar{E}_{p, q}^{k}\right)$ and $H^{i, j}\left(D_{p, q}^{k}\right):=H^{i, j}\left(\bar{D}_{p, q}^{k}\right)$, where $\bar{E}_{p, q}^{k}$ and $\bar{D}_{p, q}^{k}$ are the closures of $E_{p, q}^{k}$ and $D_{p, q}^{k}$, respectively.
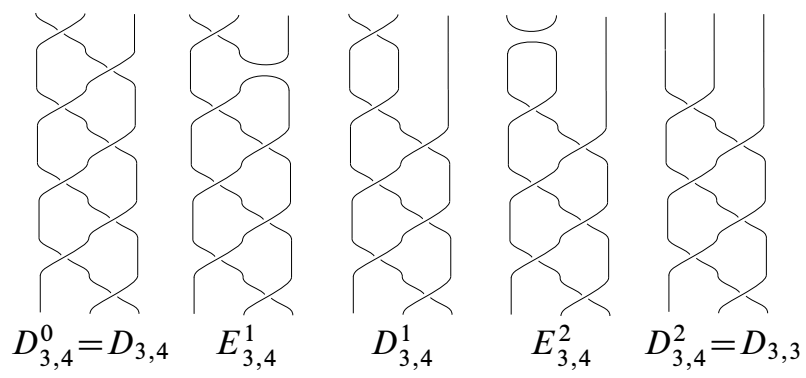

Figure 4: $D_{3,4}=D_{3,4}^{0}, E_{3,4}^{1}, D_{3,4}^{1}, E_{3,4}^{2}$ and $D_{3,4}^{2}=D_{3,3}$

From Theorem 2.2, we have the following long exact sequence for $k=1, \ldots, p-1$ :

$$
\cdots \rightarrow H^{i-1, j-1}\left(E_{p, q}^{k}\right) \rightarrow H^{i, j}\left(D_{p, q}^{k-1}\right) \rightarrow H^{i, j}\left(D_{p, q}^{k}\right) \rightarrow H^{i, j-1}\left(E_{p, q}^{k}\right) \rightarrow \cdots
$$

We use the following lemma, whose proof will be given in the appendix. 
Lemma 3.10 Let $k$ and $n$ be positive integers. Then we have

$$
H^{2 k(k+1) n}\left(D_{2 k+1,(2 k+1) n-1}\right)=0 .
$$

Proof of Lemma 3.8 To prove this lemma, it is sufficient to prove the following:

$$
\operatorname{dim}_{\mathbb{Q}} H^{2 k(k+1) n}\left(D_{2 k+1,(2 k+1) n}^{l}\right)=2\left(\begin{array}{c}
2 k+1-l \\
k+1
\end{array}\right),
$$

where $0 \leq l \leq 2 k$ (for convenience, we define $\left(\begin{array}{l}a \\ b\end{array}\right)=0$ if $0 \leq a<b$ ). Indeed, if we put $l=0$ in (3-2) then we have

$$
\begin{aligned}
\operatorname{dim}_{\mathbb{Q}} \mathrm{KH}^{2 k(k+1) n}\left(T_{2 k+1,(2 k+1) n}\right) & =\operatorname{dim}_{\mathbb{Q}} H^{2 k(k+1) n}\left(D_{2 k+1,(2 k+1) n}^{0}\right) \\
& =2\left(\begin{array}{c}
2 k+1 \\
k+1
\end{array}\right)=\left(\begin{array}{c}
2 k+2 \\
k+1
\end{array}\right) .
\end{aligned}
$$

To prove (3-2), we use induction on $k$.

For $k=1$, we need to compute $H^{4 n}\left(D_{3,3 n}\right), H^{4 n}\left(D_{3,3 n}^{1}\right)$ and $H^{4 n}\left(D_{3,3 n}^{2}\right)$. Note that $D_{3,3 n}^{2}=D_{3,3 n-1}$. The Khovanov homology of the $(3, q)$-torus link is known (for example, see [15, Theorem 8] or [16, Theorem 3.1]). In particular,

$$
\operatorname{dim}_{\mathbb{Q}} H^{4 n}\left(D_{3,3 n}^{2}\right)=\operatorname{dim}_{\mathbb{Q}} H^{4 n}\left(D_{3,3 n-1}\right)=0
$$

and

$$
\operatorname{dim}_{\mathbb{Q}} H^{4 n}\left(D_{3,3 n}^{0}\right)=\operatorname{dim}_{\mathbb{Q}} H^{4 n}\left(D_{3,3 n}\right)=6 .
$$

Next we compute the Khovanov homology of $D_{3,3 n}^{1}$. We have the following long exact sequence:

$$
\cdots \longrightarrow H^{4 n-1, j}\left(D_{3,3 n}^{2}\right) \longrightarrow H^{4 n-1, j-1}\left(E_{3,3 n}^{2}\right) \longrightarrow H^{4 n, j}\left(D_{3,3 n}^{1}\right) \longrightarrow 0 .
$$

We can check that the closure of $E_{3,3 n}^{2}$ is a diagram of the unknot and that it has $4 n-1$ negative crossings and $2 n-1$ positive crossings. From the definition of the Khovanov homology, we obtain

$$
H^{4 n-1, j-1}\left(E_{3,3 n}^{2}\right)=\mathrm{KH}^{0, j-6 n}(U)= \begin{cases}\mathbb{Q} & \text { if } j=6 n \pm 1, \\ 0 & \text { if } j \neq 6 n \pm 1,\end{cases}
$$

where $U$ is the unknot.

Hence, from (3-3), we have

$$
\operatorname{dim}_{\mathbb{Q}} H^{4 n}\left(D_{3,3 n}^{1}\right) \leq 2
$$


On the other hand, from Proposition 2.4, the dimension of $\operatorname{Lee}^{4 n}\left(D_{3,3 n}^{1}\right)$ is 2 . Since there is a spectral sequence whose $E_{\infty}$-page is the Lee homology and $E_{2}$-page is the Khovanov homology (Theorem 2.3), we have

$$
\operatorname{dim}_{\mathbb{Q}} H^{4 n}\left(D_{3,3 n}^{1}\right) \geq 2 \text {. }
$$

Hence we obtain

$$
\operatorname{dim}_{\mathbb{Q}} H^{4 n}\left(D_{3,3 n}^{1}\right)=2
$$

Suppose that (3-2) is true for $1, \ldots, k-1$, that is, suppose that for $1 \leq h<k, n>0$ and $l=0, \ldots, 2 h$, we have

$$
\operatorname{dim}_{\mathbb{Q}} H^{2 h(h+1) n}\left(D_{2 h+1,(2 h+1) n}^{l}\right)=2\left(\begin{array}{c}
2 h+1-l \\
h+1
\end{array}\right) .
$$

We will show that (3-2) is true for $k$. For $l=0, \ldots, 2 k-1$, we obtain the following long exact sequence:

$$
\begin{gathered}
\cdots \longrightarrow H^{2 k(k+1) n-1, j-1}\left(E_{2 k+1,(2 k+1) n}^{l+1}\right) \\
\stackrel{g_{j}^{l}}{\longrightarrow} H^{2 k(k+1) n, j}\left(D_{2 k+1,(2 k+1) n}^{l}\right) \\
\stackrel{f_{j}^{l}}{\longrightarrow} H^{2 k(k+1) n, j}\left(D_{2 k+1,(2 k+1) n}^{l+1}\right) \longrightarrow \cdots
\end{gathered}
$$

From the exact sequence (3-5), we obtain

$$
\begin{aligned}
\sum_{j} \operatorname{dim}_{\mathbb{Q}} & H^{2 k(k+1) n, j}\left(D_{2 k+1,(2 k+1) n}^{l}\right) \\
& \leq \sum_{j}\left(\operatorname{dim}_{\mathbb{Q}} \operatorname{Im} g_{j}^{l}+\operatorname{dim}_{\mathbb{Q}} \operatorname{Im} f_{j}^{l}\right) \\
& \leq \sum_{j}\left(\operatorname{dim}_{\mathbb{Q}} H^{2 k(k+1) n-1, j-1}\left(E_{2 k+1,(2 k+1) n}^{l+1}\right)\right. \\
& \left.\quad+\operatorname{dim}_{\mathbb{Q}} H^{2 k(k+1) n, j}\left(D_{2 k+1,(2 k+1) n}^{l+1}\right)\right) \\
& \leq \sum_{j} \sum_{m=l+1}^{2 k}\left(\operatorname{dim}_{\mathbb{Q}} H^{2 k(k+1) n-1, j-1}\left(E_{2 k+1,(2 k+1) n}^{m}\right)\right. \\
& \left.+\operatorname{dim}_{\mathbb{Q}} H^{2 k(k+1) n}\left(D_{2 k+1,(2 k+1) n}^{2 k}\right)\right) .
\end{aligned}
$$

From Lemma 3.10, we have $\operatorname{dim}_{\mathbb{Q}} H^{2 k(k+1) n}\left(D_{2 k+1,(2 k+1) n-1}\right)=0$. To compute $\operatorname{dim}_{\mathbb{Q}} H^{2 k(k+1) n-1}\left(E_{2 k+1,(2 k+1) n}^{m}\right)$, we consider the closure of $E_{2 k+1,(2 k+1) n}^{m}$. Note 
that the closure of $E_{2 k+1,(2 k+1) n}^{i}$ is equivalent to the closure of $D_{2 k-1,(2 k-1) n}^{i-2}$ for $i \geq 2$ (see Figure 5). We give the closure of $E_{2 k+1,(2 k+1) n}^{i}$ an orientation such that all crossings of the closure of $D_{2 k-1,(2 k-1) n}^{i-2}$ are positive. Then we can check that the closure of $E_{2 k+1,(2 k+1) n}^{i}$ has $4 k n-1$ negative crossings. Hence for $i \geq 2$ we have

$$
H^{2(k+1) k n-1}\left(E_{2 k+1,(2 k+1) n}^{i}\right)=\mathrm{KH}^{2(k-1) k n}\left(D_{2 k-1,(2 k-1) n}^{i-2}\right) .
$$

Similarly, the closure of $E_{2 k+1,(2 k+1) n}^{1}$ is equivalent to the closure of

$$
D_{2 k-1,(2 k-1) n} \sqcup \bigcirc,
$$

where $\bigcirc$ is a circle in the plane (see Figure 6). We give the closure of $E_{2 k+1,(2 k+1) n}^{1}$ an orientation such that all crossings of the closure of $D_{2 k-1,(2 k-1) n} \sqcup \bigcirc$ are positive. Then we can check that the closure of $E_{2 k+1,(2 k+1) n}^{1}$ also has $4 k n-1$ negative crossings. Hence we have

$$
H^{2(k+1) k n-1}\left(E_{2 k+1,(2 k+1) n}^{1}\right)=\mathrm{KH}^{2(k-1) k n}\left(D_{2 k-1,(2 k-1) n} \sqcup \bigcirc\right) .
$$
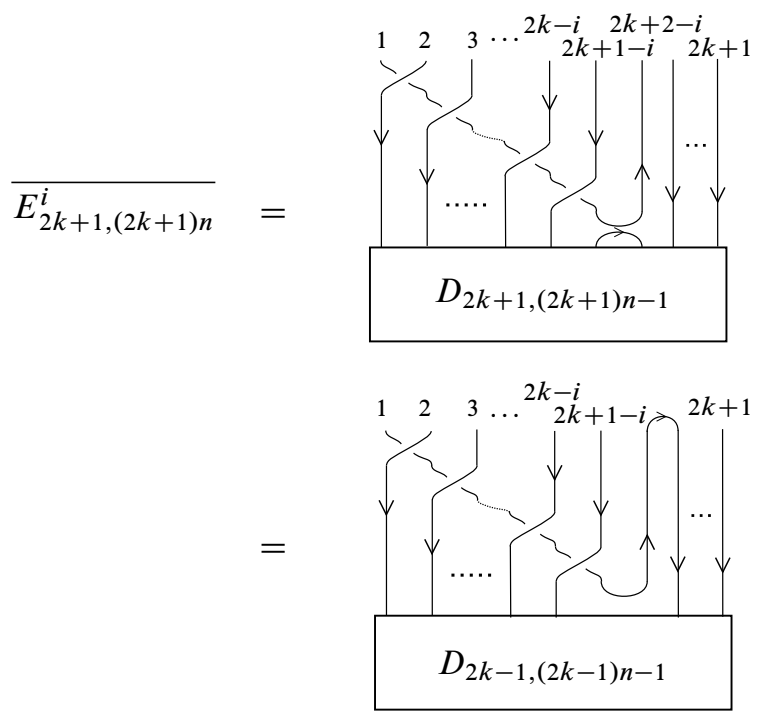

$$
=\overline{D_{2 k-1,(2 k-1) n}^{i-2}}
$$

Figure 5: The closure of $E_{2 k+1,(2 k+1) n}^{i}$ is equivalent to the closure of $D_{2 k-1,(2 k-1) n}^{i-2}$ for $i \geq 2$ 

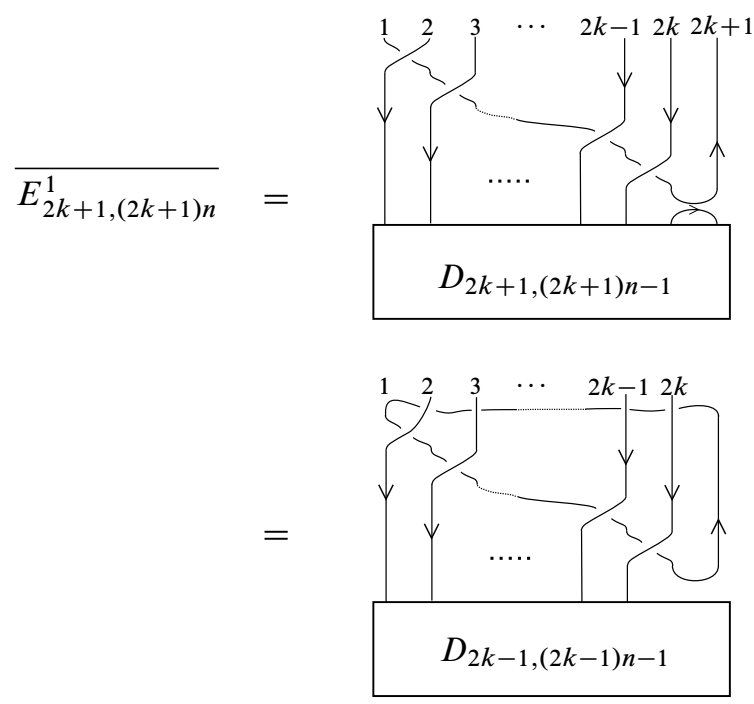

$$
=\overline{D_{2 k-1,(2 k-1) n}} \sqcup \bigcirc
$$

Figure 6: The closure of $E_{2 k+1,(2 k+1) n}^{1}$ is equivalent to the closure of $D_{2 k-1,(2 k-1) n} \sqcup \bigcirc$

By the induction hypothesis (3-4), we obtain

$$
\begin{aligned}
& \operatorname{dim}_{\mathbb{Q}} H^{2(k+1) k n-1}\left(E_{2 k+1,(2 k+1) n}^{i}\right)=2\left(\begin{array}{c}
2 k+1-i \\
k
\end{array}\right) \quad(i \geq 2), \\
& \operatorname{dim}_{\mathbb{Q}} H^{2(k+1) k n-1}\left(E_{2 k+1,(2 k+1) n}^{1}\right)=2 \times 2\left(\begin{array}{c}
2 k-1 \\
k
\end{array}\right)=2\left(\begin{array}{c}
2 k \\
k
\end{array}\right) .
\end{aligned}
$$

From (3-6), (3-7) and (3-8), we obtain

$$
\begin{aligned}
\sum_{j} \operatorname{dim}_{\mathbb{Q}} H^{2 k(k+1) n, j}\left(D_{2 k+1,(2 k+1) n}^{l}\right) & \leq \sum_{m=l+1}^{2 k} 2\left(\begin{array}{c}
2 k+1-m \\
k
\end{array}\right) \\
& =2\left(\begin{array}{c}
2 k+1-l \\
k+1
\end{array}\right) .
\end{aligned}
$$

Finally we will prove that the inequality in (3-9) is in fact an equality for $l=0, \ldots, 2 k$. We first consider the case where $l=0$. The dimension of Lee $2 k(k+1) n\left(D_{2 k+1,(2 k+1) n}\right)$ 
is $\left(\begin{array}{c}2 k+2 \\ k+1\end{array}\right)$. From Theorem 2.3, we have

$$
\begin{aligned}
\left(\begin{array}{c}
2 k+2 \\
k+1
\end{array}\right) & =\operatorname{dim}_{\mathbb{Q}} \operatorname{Lee}^{2 k(k+1) n}\left(D_{2 k+1,(2 k+1) n}\right) \\
& \leq \operatorname{dim}_{\mathbb{Q}} H^{2 k(k+1) n}\left(D_{2 k+1,(2 k+1) n}\right) \leq\left(\begin{array}{c}
2 k+2 \\
k+1
\end{array}\right) .
\end{aligned}
$$

This implies that we have the equality in (3-9) for $l=0$. Hence, for any $j \in \mathbb{Z}$ and $m=0, \ldots, 2 k-1$, the maps $g_{j}^{m}$ and $f_{j}^{m}$ in (3-5) are injective and surjective, respectively. In particular, we obtain

$$
\begin{aligned}
& \operatorname{dim}_{\mathbb{Q}} \operatorname{Im} g_{j}^{m}=\operatorname{dim}_{\mathbb{Q}} H^{2 k(k+1) n-1, j-1}\left(E_{2 k+1,(2 k+1) n}^{m+1}\right), \\
& \operatorname{dim}_{\mathbb{Q}} \operatorname{Im} f_{j}^{m}=\operatorname{dim}_{\mathbb{Q}} H^{2 k(k+1) n, j}\left(D_{2 k+1,(2 k+1) n}^{m+1}\right) .
\end{aligned}
$$

From (3-10) and (3-11), we have the equality in (3-9) for $l=0, \ldots, 2 k$ and obtain

$$
\operatorname{dim}_{\mathbb{Q}} H^{2 k(k+1) n}\left(D_{2 k+1,(2 k+1) n}^{l-1}\right)=2\left(\begin{array}{c}
2 k+2-l \\
k+1
\end{array}\right) .
$$

The following lemma can be regarded as an analog of the second claim of Theorem 3.3.

Lemma 3.11 For $i=0, \ldots, k+1$, we have

$$
\mathrm{KH}^{2 k(k+1) n, 6 k(k+1) n+1-2 i}\left(T_{2 k+1,(2 k+1) n}\right) \neq 0 .
$$

Proof To prove this lemma, we use induction on $k$.

For $k=1$, it has already known that $\mathrm{KH}^{4 n, 12 n+1}\left(T_{3,3 n}\right), \mathrm{KH}^{4 n, 12 n-1}\left(T_{3,3 n}\right)$ and $\mathrm{KH}^{4 n, 12 n-3}\left(T_{3,3 n}\right)$ are not zero (see [15, Theorem 8] or [16, Theorem 3.1]).

Suppose that Lemma 3.11 is true for $1, \ldots, k-1$, that is, suppose that for $1 \leq h<k$, $n>0$ and $i=0, \ldots, h+1$, we have

$$
\mathrm{KH}^{2 h(h+1) n, 6 h(h+1) n+1-2 i}\left(T_{2 h+1,(2 h+1) n}\right) \neq 0 .
$$

From the proof of Lemma 3.8 (recall that the inequality (3-6) is in fact an equality), we obtain

$$
\begin{aligned}
\operatorname{dim}_{\mathbb{Q}} H^{2 k(k+1) n, j}\left(D_{2 k+1,(2 k+1) n}\right) & \\
= & \sum_{m=1}^{2 k} \operatorname{dim}_{\mathbb{Q}} H^{2 k(k+1) n-1, j-1}\left(E_{2 k+1,(2 k+1) n}^{m}\right) \\
\geq & \operatorname{dim}_{\mathbb{Q}} H^{2 k(k+1) n-1, j-1}\left(E_{2 k+1,(2 k+1) n}^{1}\right) \\
& \quad+\operatorname{dim}_{\mathbb{Q}} H^{2 k(k+1) n-1, j-1}\left(E_{2 k+1,(2 k+1) n}^{2}\right) .
\end{aligned}
$$


Note that the closure of $E_{2 k+1,(2 k+1) n}^{2}$ is equivalent to the closure of $D_{2 k-1,(2 k-1) n}$ (see Figure 5). We give the closure of $E_{2 k+1,(2 k+1) n}^{2}$ an orientation such that all crossings of the closure of $D_{2 k-1,(2 k-1) n}$ are positive. Then we can check that the closure of $E_{2 k+1,(2 k+1) n}^{2}$ has $4 k n-1$ negative crossings and $2 k(2 k-1) n-1$ positive crossings. Similarly, the closure of $E_{2 k+1,(2 k+1) n}^{1}$ is equivalent to the closure of $D_{2 k-1,(2 k-1) n} \sqcup \bigcirc$, where $\bigcirc$ is a circle in the plane (see Figure 6). We give the closure of $E_{2 k+1,(2 k+1) n}^{1}$ an orientation such that all crossings of the closure of $D_{2 k-1,(2 k-1) n} \sqcup \bigcirc$ are positive. We can check that the closure of $E_{2 k+1,(2 k+1) n}^{1}$ has $4 k n-1$ negative crossings and $2 k(2 k-1) n$ positive crossings. From (3-13), we have $\operatorname{dim}_{\mathbb{Q}} \mathrm{KH}^{2 k(k+1) n, 6 k(k+1) n+1-2 i}\left(D_{2 k+1,(2 k+1) n}\right)$

$$
\begin{aligned}
& \geq \operatorname{dim}_{\mathbb{Q}} \mathrm{KH}^{2 k(k-1) n, 6 k(k-1) n+2-2 i}\left(D_{2 k-1,(2 k-1) n} \sqcup \bigcirc\right) \\
& \quad+\operatorname{dim}_{\mathbb{Q}} \mathrm{KH}^{2 k(k-1) n, 6 k(k-1) n+1-2 i}\left(D_{2 k-1,(2 k-1) n}\right) .
\end{aligned}
$$

By the induction hypothesis (3-12), the first term of the last expression is not zero for $i=1, \ldots, k+1$ and the second term is not zero for $i=0, \ldots, k$.

From Lemma 3.11, we obtain the following.

Corollary 3.12 The homological thickness of the $(2 k+1,(2 k+1) n)$-torus link is greater than or equal to $k^{2} n+2$.

Proof From Lemma 3.11, we have

$$
\mathrm{KH}^{2 k(k+1) n, 6 k(k+1) n+1-2(k+1)}\left(T_{2 k+1,(2 k+1) n}\right) \neq 0 .
$$

In [7], Khovanov determines the homological degree- 0 term of the Khovanov homology of a positive link (see Theorem 3.13 below). Note that in [7] he denotes $\mathrm{KH}^{i,-j}$ by $\mathcal{H}^{i, j}$.

The closure of $D_{2 k+1,(2 k+1) n}$ is a positive diagram of $T_{2 k+1,(2 k+1) n}$. The number of its Seifert circles is $2 k+1$ and the number of its crossings is $2 k(2 k+1) n$. From Theorem 3.13, we have

$$
\mathrm{KH}^{0,2 k((2 k+1) n-1)+1}\left(T_{2 k+1,(2 k+1) n}\right) \neq 0 .
$$

Hence, by the definition of the homological thickness (cf Corollary 3.5), we obtain

$$
\begin{aligned}
\operatorname{hw}\left(T_{2 k+1,(2 k+1) n)}\right. & \geq \frac{1}{2}(2 k((2 k+1) n-1)+1-2 k n(k+1)-1+2(k+1))+1 \\
& =k^{2} n+2 .
\end{aligned}
$$

Remark From Corollary 3.12 and Theorem 3.6, the $(2 k+1,(2 k+1) n)$-torus link has no diagram which is alternating after $k^{2} n-1$ or less crossing changes. 
Theorem 3.13 [7, Proposition 6.1] Let $L$ be a positive link. Then $\mathrm{KH}^{i}(L)=0$ if $i<0$,

$$
\mathrm{KH}^{0, j}(L)= \begin{cases}\mathbb{Q} & \text { if } j=-s_{0}(D)+c+1 \pm 1 \\ 0 & \text { otherwise }\end{cases}
$$

and $\mathrm{KH}^{i, j}(L)=0$ if $i>0$ and $j<c-s_{0}(D)$, where $s_{0}(D)$ is the number of the Seifert circles and $c$ is the number of the crossings in a positive diagram $D$ of $L$.

\section{The maximal degree of the Khovanov homology of a cable link}

In this section, we prove Theorem 1.3 and Proposition 1.4. Recall that Theorem 1.3 has three claims. These claims follow from Lemmas 4.2, 4.8 and 4.9 below, which are the first, second and third claims of Theorem 1.3, respectively. Hence, Theorem 1.3 immediately follows from these lemmas. Lemma 4.2 also implies Proposition 1.4. To prove these lemmas, we define some notations.

Definition 4.1 Let $K$ be an oriented knot and $D$ be a knot diagram of $K$ with writhe $f$. Denote the $(p, p n)$-cabling of the knot $K$ by $K(p, p n)$. Assume that each component of $K(p, p n)$ has an orientation induced by $K$, that is, each component of $K(p, p n)$ is homologous to $K$ in the tubular neighborhood of $K$. Let $D(p, q+p f)$ be the diagram depicted in Figure 7. The diagram $D(p, q+p f)$ is a diagram of the $(p, q+p f)$-cabling $K(p, q+p f)$ of $K$ (see Figure 9). Let $D^{m}(p, q+p f)$ and $E^{m}(p, q+p f)$ be the diagrams depicted in Figure 8 .

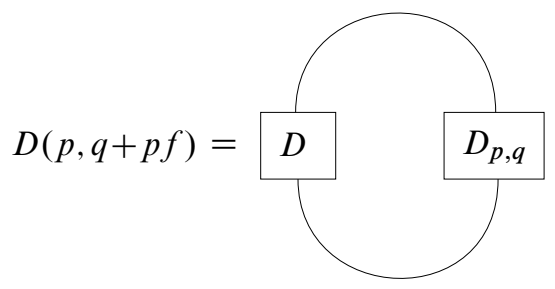

Figure 7: The diagram $D(p, q+p f)$ is obtained from $p$-parallel of $D$ by adding $D_{p, q}$, where $f$ is the writhe of $D$. The diagram $D(p, q+p f)$ is a diagram of the $(p, q+p f)$-cabling of $K$.

We first prove Lemma 4.2, which implies Corollaries 1.2 and 3.4. 


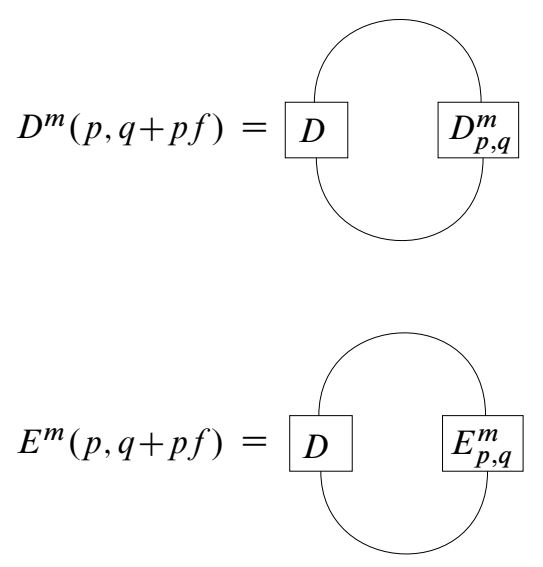

Figure 8: The diagrams $D^{m}(p, q+p f)$ and $E^{m}(p, q+p f)$

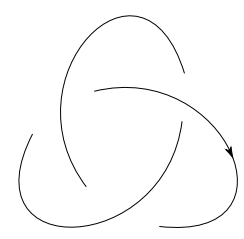

D

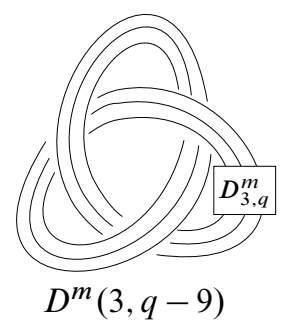

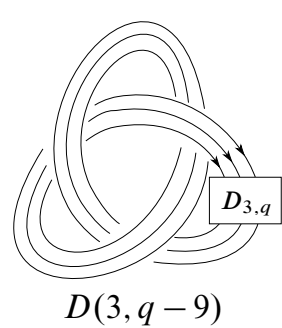

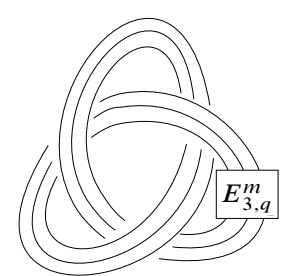

$E^{m}(3, q-9)$

Figure 9: Examples of $D(p, q), D^{m}(p, q)$ and $E^{m}(p, q)$

Lemma 4.2 Let $K$ be an oriented knot and $D$ be a diagram of $K$ with $l_{+}$positive crossings and $l_{-}$negative crossings. Put $l=l_{+}+l_{-}$and $f=l_{+}-l_{-}$. Then, for $n \geq l$ and any positive integer $k$, we have the following:

$$
\max \left\{i \in \mathbb{Z} \mid \mathrm{KH}^{i}(K(2 k, 2 k(n+f))) \neq 0\right\}=2 k^{2}(n+f)
$$

and

$$
\begin{aligned}
2 k(k+1)(n+f) & \leq \max \left\{i \in \mathbb{Z} \mid \mathrm{KH}^{i}(K(2 k+1,(2 k+1)(n+f))) \neq 0\right\} \\
& \leq 2 k(k+1)(n+f)+l_{+} .
\end{aligned}
$$


We use Lemma 4.3 below to prove Lemma 4.2. Lemma 4.3 gives upper bounds of $\max \left\{i \in \mathbb{Z} \mid \mathrm{KH}^{i}(K(p, p(n+f))) \neq 0\right\}$.

Lemma 4.3 Let $k$ be a positive integer and $n \geq 0$.

(1) If $i>2 k^{2}(n-l+1)+l(2 k)^{2}$ and $n \geq l$, or $i>l(2 k)^{2}$ and $n<l$, then we have $H^{i}\left(D^{m}(2 k, 2 k(n+f)+j)\right)=0$ for any $j=1, \ldots, 2 k$ and $m=0, \ldots, 2 k-1$.

(2) If $i>2 k(k+1)(n-l+1)+l(2 k+1)^{2}$ and $n \geq l$, or $i>l(2 k+1)^{2}$ and $n<l$, then we have $H^{i}\left(D^{m}(2 k+1,(2 k+1)(n+f)+j)=0\right.$ for any $j=1, \ldots, 2 k+1$ and $m=0, \ldots, 2 k$.

Proof of Lemma 4.3(1) We prove this by induction on $k$. For $k=1$, there is the following exact sequence:

$$
\begin{aligned}
& \cdots \longrightarrow H^{i-1}\left(E^{1}(2,2(n+f)+j)\right) \longrightarrow H^{i}(D(2,2(n+f)+j)) \longrightarrow \\
& \quad \longrightarrow H^{i}(D(2,2(n+f)+j-1)) \longrightarrow H^{i}\left(E^{1}(2,2(n+f)+j)\right) \longrightarrow \cdots
\end{aligned}
$$

where $j=1,2$ and $n \geq 0$. To study

$$
H^{i}(D(2,2(n+f)+j)) \text { and } H^{i}(D(2,2(n+f)+j-1)),
$$

we consider the diagram $E^{1}(2,2(n+f)+j)$.

Note that for $j=1,2$, the diagram $E^{1}(2,2(n+f)+j)$ is a diagram of the unknot and has $2 l+2 n+j-1$ negative crossings. Hence for $i>2 l+2 n+j-1$ and $n \geq 0$, we have $H^{i}\left(E^{1}(2,2(n+f)+j)\right)=\mathrm{KH}^{i-(2 l+2 n+j-1)}(U)=0$. From the long exact sequence (4-1), if $i>2 l+2 n+j$ and $n \geq 0$, then for $j=1,2$ we obtain

$$
H^{i}(D(2,2(n+f)+j))=H^{i}(D(2,2(n+f)+j-1)) .
$$

By repeating the same process, if $i>2 l+2 n+j$ and $n \geq 0$, then for $j=1,2$, we have

$$
\begin{aligned}
H^{i}(D(2,2(n+f)+j)) & =H^{i}(D(2,2(n+f)+j-1)) \\
& =H^{i}(D(2,2(n+f)+j-2)) \\
& =\cdots \\
& =H^{i}(D(2,2 f+1)) \\
& =H^{i}(D(2,2 f)) .
\end{aligned}
$$

Since the diagram $D(2,2 f)$ has $4 l$ crossings, we obtain $H^{i}(D(2,2 f))=0$ for any $i>4 l$. Hence if $n \geq l$ and $i>2 l+2 n+j$, or $n<l$ and $i>4 l$, then we obtain $H^{i}(D(2,2(n+f)+j))=0$, where $j=1,2$. 
Suppose that this lemma is true for $1, \ldots, k-1$, that is, suppose that for $1 \leq g<k$, $j=1, \ldots, 2 g$ and $m=0, \ldots, 2 g-1$, we have $H^{i}\left(D^{m}(2 g, 2 g(n+f)+j)\right)=0$ if $i>2 g^{2}(n-l+1)+l(2 g)^{2}$ and $n \geq l$, or $i>l(2 g)^{2}$ and $n<l$.

We will show that Lemma 4.3(1) is true for $k$. We obtain the following exact sequence:

$$
\begin{aligned}
& \rightarrow H^{i-1}\left(E^{m}(2 k, 2 k(n+f)+j)\right) \rightarrow H^{i}\left(D^{m-1}(2 k, 2 k(n+f)+j)\right) \\
& \longrightarrow H^{i}\left(D^{m}(2 k, 2 k(n+f)+j)\right) \longrightarrow H^{i}\left(E^{m}(2 k, 2 k(n+f)+j)\right) \longrightarrow,
\end{aligned}
$$

where $m=1, \ldots, 2 k-1, j=1, \ldots, 2 k$ and $n \geq 0$. We use the following claim to study $H^{i}\left(D^{m-1}(2 k, 2 k(n+f)+j)\right)$ and $H^{i}\left(D^{m}(2 k, 2 k(n+f)+j)\right)$.

Claim 4.4 Under the induction hypothesis in the proof of Lemma 4.3(1), if $i>$ $2 k^{2}(n-l+1)+l(2 k)^{2}-1$ and $n \geq l$, or $i>l(2 k)^{2}-1$ and $n<l$, then we have $H^{i}\left(E^{m}(2 k, 2 k(n+f)+j)\right)=0$ for any $j=1, \ldots, 2 k$ and $m=1, \ldots, 2 k-1$.

We will give a proof of Claim 4.4 in the appendix.

From Claim 4.4 and the exact sequence (4-2), if $i>2 k^{2}(n-l+1)+l(2 k)^{2}$ and $n \geq l$, or $i>l(2 k)^{2}$ and $n<l$, we have

$$
H^{i}\left(D^{m-1}(2 k, 2 k(n+f)+j)\right)=H^{i}\left(D^{m}(2 k, 2 k(n+f)+j)\right)
$$

for $m=1, \ldots, 2 k-1$ and $j=1, \ldots, 2 k$.

By repeating this process, if $i>2 k^{2}(n-l+1)+l(2 k)^{2}$ and $n \geq l$, or $i>l(2 k)^{2}$ and $n<l$, for $m=0, \ldots, 2 k-1$ and $j=1, \ldots, 2 k$, we have

$$
\begin{aligned}
H^{i}\left(D^{m}(2 k, 2 k(n+f)+j)\right) & =H^{i}\left(D^{m+1}(2 k, 2 k(n+f)+j)\right) \\
& =\cdots \\
& =H^{i}\left(D^{2 k-1}(2 k, 2 k(n+f)+j)\right) \\
& =H^{i}\left(D^{0}(2 k, 2 k(n+f)+j-1)\right) \\
& =H^{i}\left(D^{1}(2 k, 2 k(n+f)+j-1)\right) \\
& =\cdots \\
& =H^{i}\left(D^{2 k-1}(2 k, 2 k f+1)\right) \\
& =H^{i}(D(2 k, 2 k f))=0,
\end{aligned}
$$

where the last equality follows from the fact that the diagram $D(2 k, 2 k f)$ has $l(2 k)^{2}$ crossings. 
Proof of Lemma 4.3(2) This proof is the same as the proof of Lemma 4.3(1). We prove this by induction on $k$. For $k=1$, there is the following exact sequence:

$$
\begin{aligned}
\cdots \rightarrow & H^{i-1}\left(E^{m}(3,3(n+f)+j)\right) \rightarrow H^{i}\left(D^{m-1}(3,3(n+f)+j)\right) \longrightarrow \\
& \longrightarrow H^{i}\left(D^{m}(3,3(n+f)+j)\right) \longrightarrow H^{i}\left(E^{m}(3,3(n+f)+j)\right) \longrightarrow \cdots
\end{aligned}
$$

where $m=1,2, j=1,2,3$ and $n \geq 0$.

Note that

- $E^{1}(3,3(n+f)+1)$ is equivalent to $D$ and has $4 n+5 l_{-}+4 l_{+}$negative crossings,

- $E^{1}(3,3(n+f)+2)$ is equivalent to $D$ and has $2+4 n+5 l_{-}+4 l_{+}$negative crossings,

- $E^{1}(3,3(n+f)+3)$ is equivalent to $D \sqcup \bigcirc$ and has $3+4 n+5 l_{-}+4 l_{+}$negative crossings,

- $E^{2}(3,3(n+f)+1)$ is equivalent to $D \sqcup \bigcirc$ and has $4 n+5 l_{-}+4 l_{+}$negative crossings,

- $E^{2}(3,3(n+f)+2)$ is equivalent to $D$ and has $1+4 n+5 l_{-}+4 l_{+}$negative crossings,

- $E^{2}(3,3(n+f)+3)$ is equivalent to $D$ and has $3+4 n+5 l_{-}+4 l_{+}$negative crossings.

Hence $H^{i}\left(E^{m}(3,3(n+f)+j)\right)$ is isomorphic to $\mathrm{KH}^{i-n_{-}}(D)$ or $\mathrm{KH}^{i-n_{-}}(D \sqcup \bigcirc)$, where $n_{-}$is the number of the negative crossings of $E^{m}(3,3(n+f)+j)$. Since $D$ has only $l_{+}$positive crossings, we have $\mathrm{KH}^{i-n_{-}}(D)=\mathrm{KH}^{i-n_{-}}(D \sqcup \bigcirc)=0$ if $i-n_{-}>l_{+}$. Hence $H^{i}\left(E^{m}(3,3(n+f)+j)\right)=0$ if $i>4 n+3+5 l$ and $n \geq 0$.

From the exact sequence (4-3), if $i>4 n+4+5 l$ and $n \geq 0$, we have

$$
H^{i}\left(D^{m}(3,3(n+f)+j)\right)=H^{i}\left(D^{m-1}(3,3(n+f)+j)\right)
$$

for $j=1,2,3$ and $m=1,2$. By repeating this process, if $n \geq l$ and $i>4 n+4+5 l$, or $n<l$ and $i>9 l$, we obtain

$$
H^{i}\left(D^{m}(3,3(n+f)+j)\right)=H^{i}(D(3,3 f))=0
$$

for $j=1,2,3$ and $m=1,2$.

Suppose that this lemma is true for $1, \ldots, k-1$, that is, suppose that for $1 \leq g<k, j=$ $1, \ldots, 2 g+1$ and $m=0, \ldots, 2 g$, we have $H^{i}\left(D^{m}(2 g+1,(2 g+1)(n+f)+j)\right)=0$ 
if $i>2 g(g+1)(n-l+1)+l(2 g+1)^{2}$ and $n \geq l$, or $i>l(2 g+1)^{2}$ and $n<l$. We will show that Lemma 4.3(2) is true for $k$. We obtain the following exact sequence:

$$
\begin{aligned}
\cdots & \longrightarrow H^{i-1}\left(E^{m}(2 k+1,(2 k+1)(n+f)+j)\right) \\
& \longrightarrow H^{i}\left(D^{m-1}(2 k+1,(2 k+1)(n+f)+j)\right) \\
& \longrightarrow H^{i}\left(D^{m}(2 k+1,(2 k+1)(n+f)+j)\right) \\
& \longrightarrow H^{i}\left(E^{m}(2 k+1,(2 k+1)(n+f)+j)\right) \longrightarrow \cdots,
\end{aligned}
$$

where $m=1, \ldots, 2 k, j=1, \ldots, 2 k+1$ and $n \geq 0$. We use the following claim to study $H^{i}\left(D^{m-1}(2 k+1,(2 k+1)(n+f)+j)\right)$ and $H^{i}\left(D^{m}(2 k+1,(2 k+1)(n+f)+j)\right)$.

Claim 4.5 Under the induction hypothesis in the proof of Lemma 4.3(2), if $i>$ $2 k(k+1)(n-l+1)+l(2 k+1)^{2}-1$ and $n \geq l$, or $i>l(2 k+1)^{2}-1$ and $n<l$ then we have $H^{i}\left(E^{m}(2 k+1,(2 k+1)(n+f)+j)\right)=0$ for any $j=1, \ldots, 2 k+1$ and $m=1, \ldots, 2 k$.

We will give a proof of Claim 4.5 in the appendix.

From Claim 4.5 and the exact sequence (4-4), if $i>2 k(k+1)(n-l+1)+l(2 k+1)^{2}$ and $n \geq l$, or $i>l(2 k+1)^{2}$ and $n<l$, we have

$H^{i}\left(D^{m-1}(2 k+1,(2 k+1)(n+f)+j)\right)=H^{i}\left(D^{m}(2 k+1,(2 k+1)(n+f)+j)\right)$ for $m=1, \ldots, 2 k$ and $j=1, \ldots, 2 k+1$.

By repeating this process, if $i>2 k(k+1)(n-l+1)+l(2 k+1)^{2}$ and $n \geq l$, or $i>l(2 k+1)^{2}$ and $n<l$, then for $m=0, \ldots, 2 k$ and $j=1, \ldots, 2 k+1$, we obtain

$$
H^{i}\left(D^{m}(2 k+1,(2 k+1)(n+f)+j)\right)=H^{i}(D(2 k+1,(2 k+1) f))=0 .
$$

From Lemma 4.3, we can prove Lemma 4.2.

Proof of Lemma 4.2 From Lemma 4.3, we obtain

$$
\max \left\{i \in \mathbb{Z} \mid H^{i}(D(2 k, 2 k(n+f))) \neq 0\right\} \leq 2 k^{2}(n+l) .
$$

Hence we have

$$
\max \left\{i \in \mathbb{Z} \mid \mathrm{KH}^{i}(K(2 k, 2 k(n+f))) \neq 0\right\} \leq 2 k^{2}(n+l)-l_{-}(2 k)^{2}=2 k^{2}(n+f) .
$$

On the other hand, the dimension of $\operatorname{Lee}^{2 k^{2}(n+f)}(K(2 k, 2 k(n+f)))$ is not zero. This implies that

$$
\max \left\{i \in \mathbb{Z} \mid \mathrm{KH}^{i}(K(2 k, 2 k(n+f))) \neq 0\right\}=2 k^{2}(n+f) .
$$


Similarly we see that

$$
\max \left\{i \in \mathbb{Z} \mid \mathrm{KH}^{i}(K(2 k+1,(2 k+1)(n+f))) \leq 2 k(k+1)(n+f)+l_{+}\right.
$$

and that the dimension of $\mathrm{Lee}^{2 k(k+1)(n+f)}(K(2 k+1,(2 k+1)(n+f)))$ is not zero. Hence, we obtain

$$
\begin{aligned}
2 k(k+1)(n+f) & \leq \max \left\{i \in \mathbb{Z} \mid \mathrm{KH}^{i}(K(2 k+1,(2 k+1)(n+f))) \neq 0\right\} \\
& \leq 2 k(k+1)(n+f)+l_{+} .
\end{aligned}
$$

We use Lemma 4.6 below to prove Lemmas 4.8 and 4.9 .

Lemma 4.6 Let $K$ be a knot and $D$ be a knot diagram with $l_{+}$positive crossings and $l_{-}$negative crossings. Put $l=l_{+}+l_{-}$and $f=l_{+}-l_{-}$. For any positive integer $k$ and any $n>l$, we have $\operatorname{dim}_{\mathbb{Q}} \mathrm{KH}^{2 k^{2}(n+f)}(K(2 k, 2 k(n+f)-1))$

$$
=\operatorname{dim}_{\mathbb{Q}} H^{2 k^{2}(n+l)}(D(2 k, 2 k(n+f)-1))=0 .
$$

Proof We consider the following exact sequence:

$$
\begin{aligned}
\cdots & \longrightarrow H^{2 k^{2}(n+l)-1}\left(E^{m}(2 k, 2 k(n+f-1)+j)\right) \\
& \longrightarrow H^{2 k^{2}(n+l)}\left(D^{m-1}(2 k, 2 k(n+f-1)+j)\right) \\
& \longrightarrow H^{2 k^{2}(n+l)}\left(D^{m}(2 k, 2 k(n+f-1)+j)\right) \\
& \longrightarrow H^{2 k^{2}(n+l)}\left(E^{m}(2 k, 2 k(n+f-1)+j)\right) \longrightarrow \cdots,
\end{aligned}
$$

where $m=1, \ldots, 2 k-1, n \geq 0$ and $j=1, \ldots, 2 k-1$. To study the groups $H^{2 k^{2}(n+l)}\left(D^{m-1}(2 k, 2 k(n+f-1)+j)\right)$ and $H^{2 k^{2}(n+l)}\left(D^{m}(2 k, 2 k(n+f-1)+j)\right)$ we use the following claim.

Claim 4.7 We have $H^{i}\left(E^{m}(2 k, 2 k(n+f-1)+j)\right)=0$ if $i>l(2 k)^{2}+2 k^{2}(n-l)-2$ and $n>l$ for any $m=1, \ldots, 2 k-1$ and $j=1, \ldots, 2 k-1$.

Compare Claim 4.7 to Claim 4.4 (the main differences are the ranges of $i$ and $j$ ). We will give a proof of Claim 4.7 in the appendix.

From Claim 4.7 and the above exact sequence, if $i>l(2 k)^{2}+2 k^{2}(n-l)-1$ and $n>l$, we have

$$
H^{i}\left(D^{m-1}(2 k, 2 k(n+f-1)+j)\right)=H^{i}\left(D^{m}(2 k, 2 k(n+f-1)+j)\right),
$$


where $m=1, \ldots, 2 k-1$ and $j=1, \ldots, 2 k-1$. In particular, if $i=2 k^{2}(n+l)$, $m=1$ and $j=2 k-1$, we obtain

$$
\begin{aligned}
H^{2 k^{2}(n+l)}(D(2 k, 2 k(n+f)-1)) & =H^{2 k^{2}(n+l)}\left(D^{0}(2 k, 2 k(n+f-1)+2 k-1)\right) \\
& =H^{2 k^{2}(n+l)}\left(D^{1}(2 k, 2 k(n+f-1)+2 k-1)\right) .
\end{aligned}
$$

By repeating this process, we have

$$
\begin{aligned}
& H^{2 k^{2}(n+l)}(D(2 k, 2 k(n+f)-1))=H^{2 k^{2}(n+l)}\left(D^{1}(2 k, 2 k(n+f-1)+2 k-1)\right) \\
& =H^{2 k^{2}(n+l)}\left(D^{2}(2 k, 2 k(n+f-1)+2 k-1)\right) \\
& =\cdots \\
& =H^{2 k^{2}(n+l)}\left(D^{2 k-1}(2 k, 2 k(n+f-1)+2 k-1)\right) \\
& =H^{2 k^{2}(n+l)}\left(D^{0}(2 k, 2 k(n+f-1)+2 k-2)\right) \\
& =\cdots \\
& =H^{2 k^{2}(n+l)}(D(2 k, 2 k(n+f-1)))=0,
\end{aligned}
$$

where the last equality follows from Lemma 4.2.

By using Lemma 4.6, we will prove Lemmas 4.8 and 4.9. Lemma 4.8 is an extension of Theorem 3.3.

Lemma 4.8 Let $K$ be a knot and $D$ be a diagram of $K$ with $l_{+}$positive crossings and $l_{-}$negative crossings. Put $l=l_{+}+l_{-}$and $f=l_{+}-l_{-}$. Then for any positive integer $k$ and any $n>l$, we have

$$
\operatorname{dim}_{\mathbb{Q}} \mathrm{KH}^{2 k^{2}(n+f)}(K(2 k, 2 k(n+f)))=\left(\begin{array}{c}
2 k \\
k
\end{array}\right) .
$$

Proof As in the proof of Lemma 3.8, in order to prove this lemma, it is sufficient to prove the following:

$$
\operatorname{dim}_{\mathbb{Q}} H^{2 k^{2}(n+l)}\left(D^{i}(2 k, 2 k(n+f))\right)=2\left(\begin{array}{c}
2 k-1-i \\
k
\end{array}\right),
$$

where $0 \leq i \leq 2 k-1$ (for convenience, we define $\left(\begin{array}{l}a \\ b\end{array}\right)=0$ if $0 \leq a<b$ ). To prove (4-5), we use induction on $k$.

For $k=1$, from Lemma 4.6 we obtain $\operatorname{dim}_{\mathbb{Q}} H^{2 k^{2}(n+l)}\left(D^{1}(2,2(n+f))\right)=\operatorname{dim}_{\mathbb{Q}} H^{2 k^{2}(n+l)}(D(2,2(n+f)-1))=0$. 
Hence we have the following exact sequence:

$$
\cdots \longrightarrow H^{2(n+l)-1, j-1}\left(E^{1}(2,2(n+f))\right) \longrightarrow H^{2(n+l), j}(D(2,2(n+f))) \longrightarrow 0 .
$$

From the above exact sequence, we obtain

$$
\sum_{j} \operatorname{dim}_{\mathbb{Q}} H^{2(n+l), j}(D(2,2(n+f))) \leq \sum_{j} \operatorname{dim}_{\mathbb{Q}} H^{2(n+l)-1, j-1}\left(E^{1}(2,2(n+f))\right) .
$$

Since the diagram $E^{1}(2,2(n+f))$ is equivalent to a diagram of the unknot and has $2(n+l)-1$ negative crossings, we have

$$
\sum_{j} \operatorname{dim}_{\mathbb{Q}} H^{2(n+l)-1, j-1}\left(E^{1}(2,2(n+f))\right)=\sum_{j} \operatorname{dim}_{\mathbb{Q}} \mathrm{KH}^{0, j}(U)=2,
$$

where $U$ is the unknot. Hence we obtain

$$
\sum_{j} \operatorname{dim}_{\mathbb{Q}} H^{2(n+l), j}(D(2,2(n+f))) \leq 2 .
$$

On the other hand, the dimension of $\operatorname{Lee}^{2(n+f)}(D(2,2(n+f)))$ is 2 . Hence we obtain

$$
\operatorname{dim}_{\mathbb{Q}} H^{2(n+l)}(D(2,2(n+f)))=2 .
$$

Suppose that (4-5) is true for $1, \ldots, k-1$, that is, suppose that for $1 \leq h<k, n>0$ and $i=0, \ldots, 2 h-1$ we have

$$
\operatorname{dim}_{\mathbb{Q}} H^{2 h^{2}(n+l)}\left(D^{i}(2 h, 2 h(n+f))\right)=2\left(\begin{array}{c}
2 h-1-i \\
h
\end{array}\right) .
$$

We will show that (4-5) is true for $k$. We have the following long exact sequence:

$$
\begin{aligned}
\cdots & \longrightarrow H^{2 k^{2}(n+l)-1, j-1}\left(E^{i+1}(2 k, 2 k(n+f))\right) \\
& \stackrel{g_{j}^{i}}{\longrightarrow} H^{2 k^{2}(n+l), j}\left(D^{i}(2 k, 2 k(n+f))\right) \\
& \stackrel{f_{j}^{i}}{\longrightarrow} H^{2 k^{2}(n+l), j}\left(D^{i+1}(2 k, 2 k(n+f))\right) \longrightarrow \cdots .
\end{aligned}
$$

From the exact sequence (4-7) and the same discussion in (3-6), we obtain

$$
\begin{aligned}
& \sum_{j} \operatorname{dim}_{\mathbb{Q}} H^{2 k^{2}(n+l), j}\left(D^{i}(2 k, 2 k(n+f))\right) \\
& \leq \sum_{j} \sum_{m=i+1}^{2 k-1} \operatorname{dim}_{\mathbb{Q}} H^{2 k^{2}(n+l)-1, j-1}\left(E^{m}(2 k, 2 k(n+f))\right) \\
& \quad+\operatorname{dim}_{\mathbb{Q}} H^{2 k^{2}(n+l)(D(2 k, 2 k(n+f)-1)) .}
\end{aligned}
$$


From Lemma 4.6, we have $\operatorname{dim}_{\mathbb{Q}} H^{2 k^{2}(n+l)}(D(2 k, 2 k(n+f)-1))=0$. To compute $\operatorname{dim}_{\mathbb{Q}} H^{2 k^{2}(n+l)-1}\left(E^{m}(2 k, 2 k(n+f))\right)$, we consider $E^{m}(2 k, 2 k(n+f))$.

$E^{m}(2 k, 2 k(n+f))$ is equivalent to the diagram $D^{m-2}(2 k-2,(2 k-2)(n+f))$ for $m \geq 2$. We give $E^{m}(2 k, 2 k(n+f))$ an orientation such that all crossings of $D^{m-2}(2 k-2,(2 k-2)(n+f))$ are positive. Then $E^{m}(2 k, 2 k(n+f))$ has

$$
4 k n-2 n-1+2(2 k-1) l_{+}+\left((2 k)^{2}-2(2 k-1)\right) l_{-}
$$

negative crossings, where $l_{+}$and $l_{-}$are the number of the positive and negative crossings of $D$, respectively. Hence for $m \geq 2$ we obtain

$$
\begin{aligned}
\operatorname{dim}_{\mathbb{Q}} H^{2 k^{2}(n+l)-1} & \left(E^{m}(2 k, 2 k(n+f))\right) \\
& =\operatorname{dim}_{\mathbb{Q}} H^{2(k-1)^{2}(n+l)}\left(D^{m-2}(2 k-2,(2 k-2)(n+f))\right) \\
& =2\left(\begin{array}{c}
2 k-1-m \\
k-1
\end{array}\right) .
\end{aligned}
$$

Similarly, $E^{1}(2 k, 2 k(n+f))$ is equivalent to $D(2 k-2,(2 k-2)(n+f)) \sqcup \bigcirc$, where $\bigcirc$ is a circle in the plane. We give $E^{1}(2 k, 2 k(n+f))$ an orientation such that all crossings of $D(2 k-2,(2 k-2)(n+f)) \sqcup \bigcirc$ are positive. Then $E^{1}(2 k, 2 k(n+f))$ has $4 k n-2 n-1+2(2 k-1) l_{+}+\left((2 k)^{2}-2(2 k-1)\right) l_{-}$negative crossings. Hence we obtain

(4-10) $\operatorname{dim}_{\mathbb{Q}} H^{2 k^{2}(n+l)-1}\left(E^{m}(2 k, 2 k(n+f))\right)$

$$
\begin{aligned}
& =\operatorname{dim}_{\mathbb{Q}} H^{2(k-1)^{2}(n+l)}\left(D^{m-2}(2 k-2,(2 k-2)(n+f)) \sqcup \bigcirc\right) \\
& =2\left(\begin{array}{c}
2 k-2 \\
k-1
\end{array}\right) .
\end{aligned}
$$

From (4-8), (4-9) and (4-10), we have

$$
\begin{aligned}
& \sum_{j} \operatorname{dim}_{\mathbb{Q}} H^{2 k^{2}(n+l), j}\left(D^{i}(2 k, 2 k(n+f))\right) \\
& \leq \sum_{m=i+1}^{2 k-1} 2\left(\begin{array}{c}
2 k-1-m \\
k-1
\end{array}\right)=2\left(\begin{array}{c}
2 k-1-i \\
k
\end{array}\right) .
\end{aligned}
$$

Finally we will prove that the inequality in (4-11) is in fact an equality. At first, we consider the case where $i=0$. The dimension of $\operatorname{Lee}^{2 k^{2}(n+f)}(D(2 k, 2 k(n+f)))$ is 
$\left(\begin{array}{c}2 k \\ k\end{array}\right)$. Hence, we have

$$
\begin{aligned}
\left(\begin{array}{c}
2 k \\
k
\end{array}\right) & =\operatorname{dim}_{\mathbb{Q}} \operatorname{Lee}^{2 k^{2}(n+f)}(D(2 k, 2 k(n+f))) \\
& \leq \operatorname{dim}_{\mathbb{Q}} H^{2 k^{2}(n+l)}(D(2 k, 2 k(n+f))) \leq\left(\begin{array}{c}
2 k \\
k
\end{array}\right) .
\end{aligned}
$$

This implies that we have the equality in (4-11) for $i=0$. This fact implies that for any $j \in \mathbb{Z}$ and $m=0, \ldots, 2 k-2$, the maps $g_{j}^{m}$ and $f_{j}^{m}$ in (4-7) are injective and surjective, respectively. Hence, we have the equality in (4-11) for $i=0, \ldots, 2 k-1$ and we obtain

$$
\begin{aligned}
\operatorname{dim}_{\mathbb{Q}} H^{2 k^{2}(n+l)}\left(D^{i}(2 k, 2 k(n+f))\right) & =\sum_{j} \operatorname{dim}_{\mathbb{Q}} H^{2 k^{2}(n+l), j}\left(D^{i}(2 k, 2 k(n+f))\right) \\
& =2\left(\begin{array}{c}
2 k-1-i \\
k
\end{array}\right) .
\end{aligned}
$$

Next we prove Lemma 4.9.

Lemma 4.9 Let $K$ be a knot and $D$ be a diagram of $K$ with $l_{+}$positive crossings and $l_{-}$negative crossings. Put $l=l_{+}+l_{-}$and $f=l_{+}-l_{-}$. Then for any $n>l$, any positive integer $k$ and $i=0, \ldots, k$, we have

$$
\mathrm{KH}^{2 k^{2}(n+f), 6 k^{2}(n+f)-2 i}(K(2 k, 2 k(n+f))) \neq 0 .
$$

Proof We use induction on $k$. In the case where $k=1$, we need to prove

$$
\mathrm{KH}^{2(n+f), 6(n+f)-1 \pm 1}(D(2,2(n+f))) \neq 0 .
$$

We have the exact sequence

$$
\begin{aligned}
\cdots \longrightarrow H^{2(n+l)-1, j-1}\left(E^{1}(2,2(n+f))\right) & \longrightarrow H^{2(n+l), j}(D(2,2(n+f))) \\
& \longrightarrow H^{2(n+l), j}\left(D^{1}(2,2(n+f))\right) \longrightarrow \cdots .
\end{aligned}
$$

It follows from Lemma 4.6 that

$$
H^{2(n+l), j}\left(D^{1}(2,2(n+f))\right)=H^{2(n+l), j}(D(2,2(n+f)-1))=0 .
$$

The diagram $E^{1}(2,2(n+f))$ is equivalent to a diagram of the unknot and has $2 l$ positive crossings and $2 l+2 n-1$ negative crossings. Hence we have

$$
H^{2(n+l)-1, j-1}\left(E^{1}(2,2(n+f))\right)= \begin{cases}\mathbb{Q} & \text { if } j=2 l+4 n-1 \pm 1 \\ 0 & \text { otherwise }\end{cases}
$$


By Lemma 4.8, we have $\operatorname{dim}_{\mathbb{Q}} H^{2(n+l)}(D(2,2(n+f)))=2$. From the above exact sequence, we have $H^{2(n+l)-1, j-1}\left(E^{1}(2,2(n+f))\right)=H^{2(n+l), j}(D(2,2(n+f)))$ since $\operatorname{dim}_{\mathbb{Q}} H^{2(n+l)}(D(2,2(n+f)))=2=\operatorname{dim}_{\mathbb{Q}} H^{2(n+l)-1}\left(E^{1}(2,2(n+f))\right)$. Hence we obtain

$$
\begin{aligned}
\mathrm{KH}^{2(n+f), 6(n+f)-1 \pm 1}(D(2,2(n+f))) & =H^{2(n+l), 2 l+4 n-1 \pm 1}(D(2,2(n+f))) \\
& =H^{2(n+l)-1,2 l+4 n-2 \pm 1}\left(E^{1}(2,2(n+f))\right) \\
& =\mathbb{Q} .
\end{aligned}
$$

Suppose that Lemma 4.9 is true for $1, \ldots, k-1$, that is, suppose that for $1 \leq h<k$, $n>0$ and $i=0, \ldots, h$, we have

$$
\mathrm{KH}^{2 h^{2}(n+f), 6 h^{2}(n+f)-2 i}(K(2 h, 2 h(n+f))) \neq 0 .
$$

From the proof of Lemma 4.8 (, recall that the inequality (4-8) is in fact an equality), we have

$$
\begin{aligned}
& \operatorname{dim}_{\mathbb{Q}} H^{2 k^{2}(n+l), j}(D(2 k, 2 k(n+f))) \\
& \geq \operatorname{dim}_{\mathbb{Q}} H^{2 k^{2}(n+l)-1, j-1}\left(E^{1}(2 k, 2 k(n+f))\right) \\
& \quad+\operatorname{dim}_{\mathbb{Q}} H^{2 k^{2}(n+l)-1, j-1}\left(E^{2}(2 k, 2 k(n+f))\right) .
\end{aligned}
$$

The diagram $E^{1}(2 k, 2 k(n+f))$ is equivalent to $D(2 k-2,(2 k-2)(n+f)) \sqcup \bigcirc$, where $\bigcirc$ is a circle in the plane. We give $E^{1}(2 k, 2 k(n+f))$ an orientation such that all crossings of $D(2 k-2,(2 k-2)(n+f)) \sqcup \bigcirc$ are positive. Then $E^{1}(2 k, 2 k(n+f))$ has $2(2 k-1)(f+n)-1+l_{-}(2 k)^{2}$ negative crossings and $(2 k)^{2} l+(2 k-1) 2 k n-1$ crossings. Similarly, the diagram $E^{2}(2 k, 2 k(n+f))$ is equivalent to the diagram $D(2 k-2,(2 k-2)(n+f))$. We give $E^{2}(2 k, 2 k(n+f))$ an orientation such that all crossings of $D(2 k-2,(2 k-2)(n+f))$ are positive. Then $E^{2}(2 k, 2 k(n+f))$ has $2(2 k-1)(f+n)-1+l_{-}(2 k)^{2}$ negative crossings and $(2 k)^{2} l+(2 k-1) 2 k n-2$ crossings. From (4-13), we have

$$
\begin{aligned}
\operatorname{dim}_{\mathbb{Q}} & \mathrm{KH}^{2 k^{2}(n+f), 6 k^{2}(n+f)-2 i}(D(2 k, 2 k(n+f))) \\
\geq \operatorname{dim}_{\mathbb{Q}} & \mathrm{KH}^{2(k-1)^{2}(n+f), 6(k-1)^{2}(n+f)-2 i+1}(D(2 k-2,(2 k-2)(n+f)) \sqcup \bigcirc) \\
\quad & +\operatorname{dim}_{\mathbb{Q}} \mathrm{KH}^{2(k-1)^{2}(n+f), 6(k-1)^{2}(n+f)-2 i}(D(2 k-2,(2 k-2)(n+f))) .
\end{aligned}
$$

By the induction hypothesis (4-12), the first term of the last expression is not zero for $i=1, \ldots, k$, and the second term is not zero for $i=0, \ldots, k-1$. This completes the proof. 
Remark In general Lemma 4.6 is not true for $(2 k+1,(2 k+1) n)$-cable links, that is, $\operatorname{dim}_{\mathbb{Q}} \mathrm{KH}^{2 k(k+1)(n+f)}(D(2 k+1,(2 k+1)(n+f)-1)) \neq 0$ even though $n>l$. The reason is that the maximal homological degree of the Khovanov homology of a $(2 k+1,(2 k+1) n)$-cable link is not equal to that of the Lee homology of the link. Since we need Lemma 4.6 to prove Lemmas 4.8 and 4.9, we cannot obtain results for $(2 k+1,(2 k+1) n)$-cable links corresponding to these lemmas by the same methods.

From Lemma 4.9, we obtain the following.

Corollary 4.10 Let $K$ be a positive knot and $D$ be a positive diagram of $K$ with $l$ crossings. Then for any $n>l$ and any positive integer $k$, the homological thickness $\mathrm{hw}(K(2 k, 2 k(n+l)))$ is greater than or equal to $k(k-1)(n+l)+2+k s(K)$, where $s(K)$ is the Rasmussen invariant of $K$.

Proof By Lemma 4.9, we have

$$
\mathrm{KH}^{2 k^{2}(n+l), 6 k^{2}(n+l)-2 k}(K(2 k, 2 k(n+l))) \neq 0 .
$$

Since $D(2 k, 2 k(n+l))$ is also positive diagram, from Theorem 3.13, we obtain

$$
\mathrm{KH}^{0,4 k^{2} l+2 k n(2 k-1)-2 k s_{0}(D)+2}(K(2 k, 2 k(n+l))) \neq 0,
$$

where $s_{0}(D)$ is the number of Seifert circles of $D$. Hence

$$
\operatorname{hw}(K(2 k, 2 k(n+l))) \geq k(k-1)(n+l)+2+k\left(l+1-s_{0}(D)\right) .
$$

It is known that the Rasmussen invariant $s(K)$ of a positive knot $K$ is $l+1-s_{0}(D)$, where $D$ is a positive diagram of $K$ with $l$ crossings (see [13, Section 5.2]). Hence we obtain

$$
\operatorname{hw}(K(2 k, 2 k(n+l))) \geq k(k-1)(n+l)+2+k \cdot s(K) .
$$

Remark Corollary 4.10 is an extension of Corollary 3.5. From Theorem 3.6, if $n$ is sufficiently large, the $(2 k, 2 k n)$-cabling of any positive knot $K$ has no diagram which is alternating after $k(k-1) n+k s(K)-1$ or less crossing changes.

\section{An application for twisted Whitehead doubles}

In this section, we consider twisted Whitehead doubles of any knot and compute their Khovanov homologies. 
Let $K$ be a knot. A twisted Whitehead double of $K$ is represented by the diagram $L(D, q)$ in Figure 10, where $D$ is a diagram of $K$ and $q$ is an integer. The right picture in Figure 11 is a twisted Whitehead double of the left-handed trefoil.

A cable link is obtained from a twisted Whitehead double of any knot by smoothing at a crossing. In Section 4, we give some computations of the Khovanov homology groups of cable links. By applying these computations, we will calculate the Khovanov homology groups of a twisted Whitehead double of any knot with sufficiently many twists. Moreover we compute their Rasmussen invariants (Corollary 5.6).

Let $D$ be a knot diagram with $l_{+}(D)$ positive crossings and $l_{-}(D)$ negative crossings. Put $l=l_{+}(D)+l_{-}(D)$ and $f=l_{+}(D)-l_{-}(D)$. Let $L(D, q)=L, L_{0}$ and $L_{1}$ be knot diagrams depicted in Figure 10, where $q$ is a nonnegative integer (for example, see Figure 11). In the case where $q$ is negative, we define $L(D, q)$ as the mirror image of $L(-D,-q+1)$, where $-D$ is the mirror image of $D$.

By the definition, we have

$$
\begin{aligned}
& H^{i, j}\left(L_{1}\right)=H^{i-1, j-2}(D(2, q+2 f)), \\
& H^{i, j}\left(L_{0}\right)=H^{i-1, j-1}(D(2, q-1+2 f)) .
\end{aligned}
$$

To study the Khovanov homology of $L(D, q)$, we compute $H^{i, j}(D(2, q-1+2 f))$ for some $i$ and $j$.

Lemma 5.1 For $n>l+1$, we have

$$
H^{2(n+l)-1, j}(D(2,2(n+f)-1))= \begin{cases}\mathbb{Q} & \text { if } j=2 l+4 n-2, \\ 0 & \text { if } j \neq 2 l+4 n-3 \pm 1,\end{cases}
$$

and for $n>l$ and any $i \geq 2(n+l)$, we have

$$
H^{i}(D(2,2(n+f)-1))=0 .
$$

Proof We obtain the following exact sequence:

$$
\begin{aligned}
\cdots & \longrightarrow H^{2(n+l)-2, j}\left(D^{1}(2,2(n+f)-1)\right) \\
& \longrightarrow H^{2(n+l)-2, j-1}\left(E^{1}(2,2(n+f)-1)\right) \\
& \longrightarrow H^{2(n+l)-1, j}(D(2,2(n+f)-1)) \\
& \longrightarrow H^{2(n+l)-1, j}\left(D^{1}(2,2(n+f)-1)\right) \longrightarrow \cdots,
\end{aligned}
$$

where $E^{m}(p, q)$ and $D^{m}(p, q)$ are given in Figure 8. By Lemma 4.2 we have

$$
H^{2(n+l)-1, j}\left(D^{1}(2,2(n+f)-1)\right)=H^{2(n+l)-1, j}(D(2,2(n+f)-2))=0 .
$$



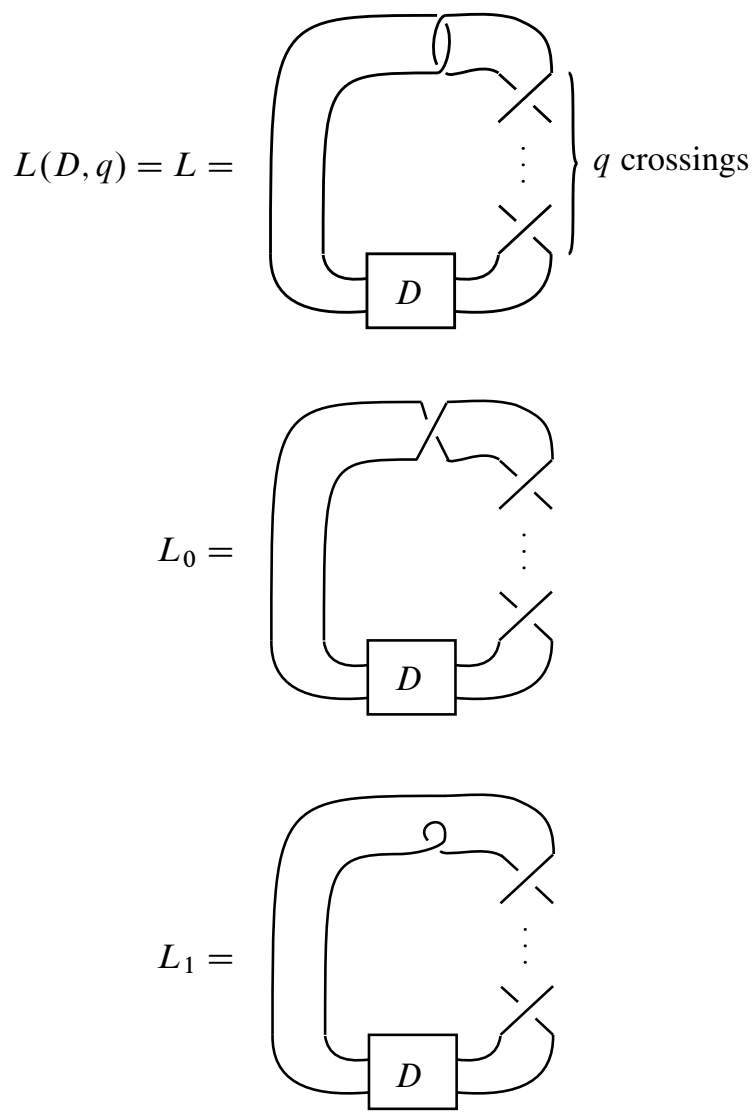

Figure 10: $L(D, q)=L, L_{0}$ and $L_{1}$, where $q$ is nonnegative
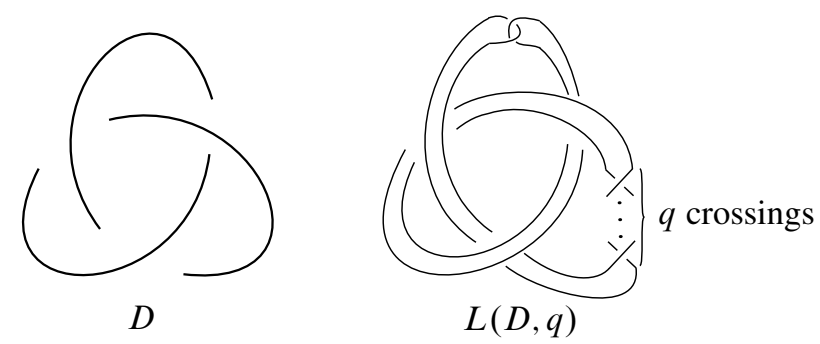

Figure 11: An example of $L(D, q)$

The diagram $E^{1}(2,2(n+f)-1)$ is a diagram of the unknot and has $2 l+2 n-2$ negative crossings and $2 l$ positive crossings. Hence we have

$$
H^{2(n+l)-2, j-1}\left(E^{1}(2,2(n+f)-1)\right)= \begin{cases}\mathbb{Q} & \text { if } j=2 l+4 n-3 \pm 1, \\ 0 & \text { otherwise. }\end{cases}
$$


By Lemmas 4.9 and 4.8, we obtain

$$
H^{2(n+l)-2, j}(D(2,2(n+f)-2))= \begin{cases}\mathbb{Q} & \text { if } j=2 l+4 n-5 \pm 1, \\ 0 & \text { otherwise. }\end{cases}
$$

From the above exact sequence, we have

$$
H^{2(n+l)-1, j}(D(2,2(n+f)-1))= \begin{cases}\mathbb{Q} & \text { if } j=2 l+4 n-2, \\ 0 & \text { if } j \neq 2 l+4 n-3 \pm 1 .\end{cases}
$$

The second claim follows from Lemmas 4.6 and 4.2.

By using Lemma 5.1, we can compute some Khovanov homology groups of $L(D, q)$.

Proposition 5.2 Let $D$ be a knot diagram with $l_{+}(D)$ positive crossings and $l_{-}(D)$ negative crossings. Put $l=l_{+}(D)+l_{-}(D)$. Let $n$ be an integer which is greater than $l$.

(I) In the case where $q=2 n$, we have

$$
\mathrm{KH}^{0, j}(L(D, q))= \begin{cases}\mathbb{Q} & \text { if } j=-2 \pm 1 \\ 0 & \text { otherwise. }\end{cases}
$$

(II) In the case where $q=2 n+1$, we have

$$
\mathrm{KH}^{2, j}(L(D, q))= \begin{cases}\mathbb{Q} & \text { if } j=5 \\ 0 & \text { if } j \neq 5,3\end{cases}
$$

Proof Put $f=l_{+}(D)-l_{-}(D)$.

(I) Suppose that $q=2 n$.

From Lemma 4.8, we obtain $\operatorname{dim}_{\mathbb{Q}} H^{2(n+l)}(D(2,2(f+n)))=2$. From Lemma 4.9, we have $H^{2(n+l), 4 n+2 l-1 \pm 1}(D(2,2(f+n))) \neq 0$. Hence we obtain

$$
H^{2(n+l)+1, j}\left(L_{1}\right)=H^{2(n+l), j-2}(D(2,2(f+n)))= \begin{cases}\mathbb{Q} & \text { if } j=4 n+2 l+1 \pm 1, \\ 0 & \text { otherwise. }\end{cases}
$$

From Lemma 5.1, we obtain $H^{i, j}\left(L_{0}\right)=H^{i-1, j-1}(D(2,2(f+n)-1))=0$ if $i>2(n+l)$. Now there is the following exact sequence:

$$
\begin{aligned}
\cdots \longrightarrow H^{2(n+l)+1, j}\left(L_{0}\right) & \longrightarrow H^{2(n+l)+1, j-1}\left(L_{1}\right) \\
& \longrightarrow H^{2(n+l)+2, j}(L) \longrightarrow H^{2(n+l)+2, j}\left(L_{0}\right) \longrightarrow \cdots .
\end{aligned}
$$

Since $H^{2(n+l)+1, j}\left(L_{0}\right)=H^{2(n+l)+2, j}\left(L_{0}\right)=0$, we have

$$
H^{2(n+l)+2, j}(L)= \begin{cases}\mathbb{Q} & \text { if } j=4 n+2 l+2 \pm 1, \\ 0 & \text { otherwise. }\end{cases}
$$


The diagram $L=L(D, 2 n)$ has $2 n+2+2 l$ negative crossings and $2 l$ positive crossings. By the definition, we obtain

$$
\mathrm{KH}^{0, j}(L(D, q))= \begin{cases}\mathbb{Q} & \text { if } j=-2 \pm 1 \\ 0 & \text { otherwise }\end{cases}
$$

(II) Suppose that $q=2 n+1$.

We can prove this by the same method as in (I). It follows from Lemmas 4.2 and 5.1 that

$$
\begin{aligned}
H^{2(n+l)+2, j}\left(L_{1}\right) & =H^{2(n+l)+1, j-2}(D(2,2 f+2 n+1)) \\
& = \begin{cases}\mathbb{Q} & \text { if } j=4 n+2 l+4, \\
0 & \text { if } j \neq 4 n+2 l+3 \pm 1,\end{cases}
\end{aligned}
$$

and $H^{i, j}\left(L_{0}\right)=H^{i-1, j-1}(D(2,2 f+2 n))=0$ if $i>2(n+l)+1$. Now we have the following exact sequence:

$$
H^{2(n+l)+2, j}\left(L_{0}\right) \rightarrow H^{2(n+l)+2, j-1}\left(L_{1}\right) \rightarrow H^{2(n+l)+3, j}(L) \rightarrow H^{2(n+l)+3, j}\left(L_{0}\right) .
$$

Since $H^{2(n+l)+2, j}\left(L_{0}\right)=H^{2(n+l)+3, j}\left(L_{0}\right)=0$, we obtain

$$
H^{2(n+l)+3, j}(L)=H^{2(n+l)+2, j-1}\left(L_{1}\right)= \begin{cases}\mathbb{Q} & \text { if } j=4 n+2 l+5, \\ 0 & \text { if } j \neq 4 n+2 l+4 \pm 1 .\end{cases}
$$

The diagram $L=L(D, 2 n+1)$ has $2 n+1+2 l$ negative crossings and $2+2 l$ positive crossings. By the definition we have

$$
\mathrm{KH}^{2, j}(L(D, q))= \begin{cases}\mathbb{Q} & \text { if } j=5 \\ 0 & \text { if } j \neq 5,3\end{cases}
$$

Corollary 5.3 Let $D$ be a knot diagram with $l_{+}(D)$ positive crossings and $l_{-}(D)$ negative crossings. Put $l=l_{+}(D)+l_{-}(D)$. Let $n$ be an integer which is greater than $l$. Then we have $s(L(D, 2 n))=-2$, where $s(K)$ is the Rasmussen invariant of a knot $K$.

Proof From Proposition 2.4, we have $\operatorname{dim}_{\mathbb{Q}} \operatorname{Lee}^{0}(L(D, 2 n))=2$. Let $s_{\max }$ and $s_{\min }$ be its generators. Assume that the $q$-grading of $s_{\max }$ is greater than that of $s_{\min }$. From the definition of the Rasmussen invariant, the $q$-grading of $s_{\max }$ is $s(L(D, 2 n))+1$ and that of $s_{\min }$ is $s(L(D, 2 n))-1$. Since there is a spectral sequence whose $E_{\infty}$-page is the Lee homology and $E_{2}$-page is the Khovanov homology, we have

$$
\mathrm{KH}^{0, s(L(D, 2 n)) \pm 1}(L(D, 2 n)) \neq 0 .
$$

From Proposition 5.2(I), we have $s(L(D, 2 n))=-2$. 
In [12] Livingston and Naik showed Theorem 5.5 below, which gives a relation between the values of the Rasmussen invariants of $L(D, 2 t)$ and $L(D, 2 t+1)$.

Definition 5.4 We call an invariant $v$ of Livingston-Naik type if $v$ is an integer-valued additive knot invariant which bounds the smooth 4-genus of a knot and coincides with the 4-ball genera of positive torus knots, that is:

- $\quad v$ is a homomorphism from the smooth knot concordance group $\mathcal{C}$ to $\mathbb{Z}$.

- $|v(K)| \leq g_{4}(K)$, where $g_{4}(K)$ is the 4-genus of a knot $K$.

- $v\left(T_{p, q}\right)=(p-1)(q-1) / 2$, where $p$ and $q$ are coprime integers.

Remark For example the Ozsváth-Szabó invariant $\tau$ and half of the Rasmussen invariant $s / 2$ are Livingston-Naik-type invariants.

Theorem 5.5 [12, Theorem 1] Let $v$ be a Livingston-Naik type invariant. If $v(L(D, 2 t))= \pm 1$, then $v(L(D, 2 t+1))=0$.

Remark In their paper, Livingston and Naik use the notation $D_{-}(K, t)$ and $D_{+}(K, t)$ instead of $L(D, 2 t-2 f)$ and $L(D, 2 t+1-2 f)$ respectively.

Theorem 5.5 does not determine the value of the Rasmussen invariant of a twisted Whitehead double of a knot. From Theorem 5.5 and Corollary 5.3, we can compute the Rasmussen invariants of twisted Whitehead doubles of any knot with sufficiently many twists.

Corollary 5.6 For any $n>l$, we have

$$
\begin{aligned}
s(L(D, 2 n)) & =-2, \\
s(L(D, 2 n+1)) & =0, \\
s(L(D,-2 n)) & =0, \\
s(L(D,-2 n+1)) & =2 .
\end{aligned}
$$

Proof Let $-D$ be the mirror image of the diagram $D$. From Proposition 5.2, we have $s(L(D, 2 n))=-2$. Since $L(D,-2 n+1)$ and the mirror image of $L(-D, 2 n)$ are diagrams of the same knot, we obtain $s(L(D,-2 n+1))=-s(L(-D, 2 n))$. Since we can apply Proposition 5.2 to $L(-D, 2 n)$, we have $s(L(D,-2 n+1))=$ $-s(L(-D, 2 n))=2$. It follows from Theorem 5.5 that $s(L(D, 2 n+1))=0=$ $s(L(-D, 2 n+1))$. Since $L(D,-2 n)$ and the mirror image of $L(-D, 2 n+1)$ are diagrams of the same knot, we have $s(L(D,-2 n))=0$. 
We can rewrite Corollary 5.6 as follows.

Corollary 5.7 For any knot $K$, we have $s\left(D_{+}(K, t)\right)=0$ for $t>2 l_{+}(K)$ and $s\left(D_{+}(K, t)\right)=2$ for $t<-2 l_{-}(K)$, where $l_{+}(K)=\min \left\{l_{+}(D) \mid D\right.$ is a diagram of $\left.K\right\}$ and $l_{-}(K)=\min \left\{l_{-}(D) \mid D\right.$ is a diagram of $\left.K\right\}$ (see Figure 12).

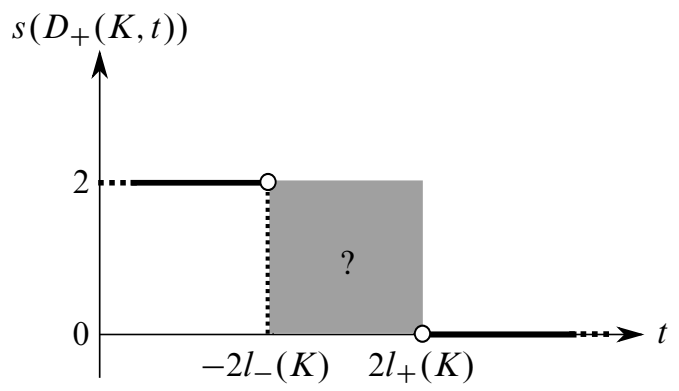

Figure 12: $s\left(D_{+}(K, t)\right)$

Remark Note that we use a relation between the Khovanov homology and the Rasmussen invariant $s$ in Corollary 5.7 (or Corollary 5.6). We do not know whether another Livingston-Naik-type invariant satisfies Corollary 5.7 or not.

We only compute the Khovanov homology groups of a twisted Whitehead double of any knot with sufficiently many twists. Since the Rasmussen invariant $s$ is obtained from the Lee homology, the estimation in Corollary 5.7 may not be sharp. Livingston and Naik [12] showed the following theorem, which is similar to Corollary 5.7.

Theorem 5.8 [12, Theorem 2] Let $v$ be a Livingston-Naik-type invariant. For each knot $K$, we have $v\left(D_{+}(K, t)\right)=1$ for $t \leq \mathrm{TB}(K)$ and $v\left(D_{+}(K, t)\right)=0$ for $t \geq-\mathrm{TB}(-K)$, where $\mathrm{TB}(K)$ is the maximal Thurston-Bennequin number of a knot $K$ and $-K$ is the mirror image of $K$.

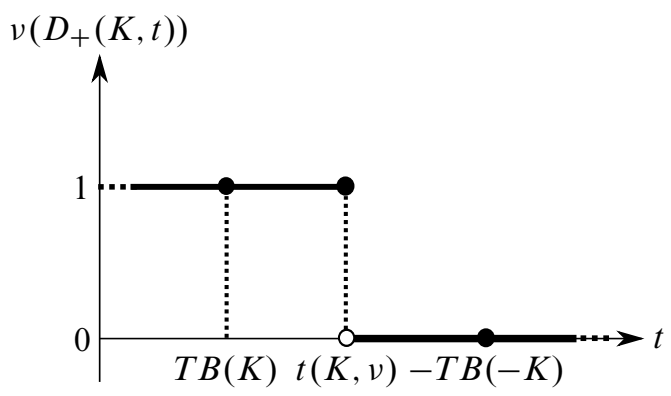

Figure 13: $v\left(D_{+}(K, t)\right)$ 


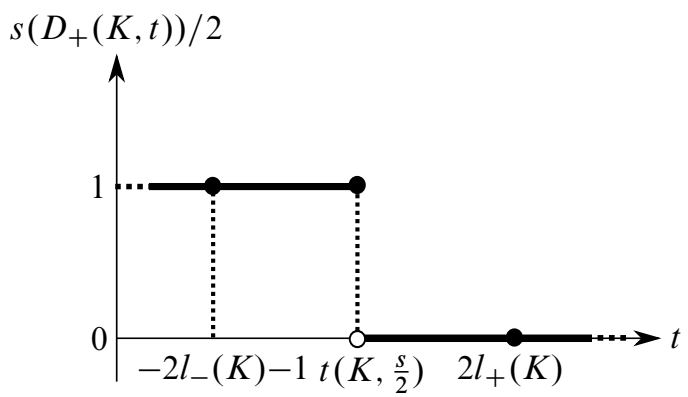

Figure 14: $s\left(D_{+}(K, t)\right) / 2$

Remark For any Livingston-Naik-type invariant $v$ and knot $K$, Livingston and Naik show that $v\left(D_{+}(K, t)\right)$ is a nonincreasing function of $t$. Hence, there exists an integer $t(K, v)$ such that $v\left(D_{+}(K, t)\right)=1$ for $t \leq t(K, v)$ and $v\left(D_{+}(K, t)\right)=0$ for $t>t(K, v)$ (see [12, Theorem 2]).

For any Livingston-Naik-type invariant $v$, we have $T B(K) \leq t(K, v)<-T B(-K)$ from Theorem 5.8 (Figure 13). In particular, we obtain

$$
T B(K) \leq t(K, s / 2)<-T B(-K) .
$$

From Corollary 5.7, we have

$$
-2 l_{-}(K)-1 \leq t(K, s / 2) \leq 2 l_{+}(K) .
$$

See also Figure 14. As far as the author knows, there is no relation between the maximal Thurston-Bennequin number and the positive or negative crossing number. However they have a similar property as above.

For the Ozsváth-Szabó invariant $\tau$, it is known that $t(K, \tau)=2 \tau(K)-1$ (see Theorem 5.10 below).

Example 5.9 For the right-handed trefoil $T_{2,3}$, we have $l_{-}\left(T_{2,3}\right)=0, l_{+}\left(T_{2,3}\right)=3$, $\operatorname{TB}\left(T_{2,3}\right)=1$ and $\operatorname{TB}\left(-T_{2,3}\right)=-6$. We have $s\left(D_{+}\left(T_{2,3}, t\right)\right)=2$ for $t \leq 1$ and $s\left(D_{+}\left(T_{2,3}, t\right)\right)=0$ for $t \geq 6$ from Theorem 5.8. From Corollary 5.7, we have $s\left(D_{+}\left(T_{2,3}, t\right)\right)=2$ for $t \leq 1$ and $s\left(D_{+}\left(T_{2,3}, t\right)\right)=0$ for $t \geq 7$. Hence, in this case, Theorem 5.8 implies Corollary 5.7. However, in general, we do not know whether Theorem 5.8 implies Corollary 5.7 or not.

Theorem 5.10 [5, Theorem 1.4] For any knot $K$, we have

$$
\tau\left(D_{+}(K, t)\right)= \begin{cases}0 & \text { if } t>2 \tau(K)-1 \\ 1 & \text { if } t \leq 2 \tau(K)-1 .\end{cases}
$$


Remark The negative half of the knot signature $-\sigma / 2$ is not of a Livingston-Naik type since $-\sigma\left(T_{p, q}\right) / 2$ is not equal to $(p-1)(q-1) / 2$. However it has similar properties. We call such an invariant of weak Livingston-Naik-type (see Definition 5.11 below).

Definition 5.11 We call an invariant $v^{\prime}$ of weak Livingston-Naik-type if $v^{\prime}$ is an integer-valued additive knot invariant which bounds the smooth 4-genus of a knot and coincides with the 4-ball genus of right-handed trefoil knot, that is:

- $v^{\prime}$ is a homomorphism from the smooth knot concordance group $\mathcal{C}$ to $\mathbb{Z}$.

- $\left|v^{\prime}(K)\right| \leq g_{4}(K)$, where $g_{4}(K)$ is the 4-genus of a knot $K$.

- $v^{\prime}\left(T_{2,3}\right)=1$.

Remark In [1], Abe calls the properties in Definition 5.11 the L-property.

Remark For any Livingston-Naik-type invariant $v$, we only use the properties in Definition 5.11 to prove that $v\left(D_{+}(K, t)\right)$ is a nonincreasing function of $t$. Hence, for any weak Livingston-Naik-type invariant $v^{\prime}$ and knot $K, v^{\prime}\left(D_{+}(K, t)\right)$ is a nonincreasing function of $t$ and there exists an integer $t\left(K, v^{\prime}\right)$ such that $v^{\prime}\left(D_{+}(K, t)\right)=1$ for $t \leq t\left(K, v^{\prime}\right)$ and $v^{\prime}\left(D_{+}(K, t)\right)=0$ for $t>t\left(K, v^{\prime}\right)$ (see [12, Theorem 2] and [11, Corollary 3]). In particular, the negative half of the knot signature $\sigma$ is of weak Livingston-Naik-type and $t(K,-\sigma / 2)=0$.

\section{Appendix}

In this section, we prove Claims 4.4, 4.5 and 4.7 and Lemma 3.10.

Proof of Claim 4.4 We consider the diagram $E^{m}(2 k, 2 k(n+f)+j)$. If we slide an arc (which is like a "cap" illustrated in the following figures) of $E^{m}(2 k, 2 k(n+f)+j)$, the diagram $E^{m}(2 k, 2 k(n+f)+j)$ may change to one of the four diagrams depicted in Figures 15, 16, 17 and 18. If $E^{m}(2 k, 2 k(n+f)+j)$ changes to the diagram depicted in Figure 17, then we continue the isotopic moves as depicted in Figure 19. Similarly, if $E^{m}(2 k, 2 k(n+f)+j)$ changes to the diagram depicted in Figure 18, then we continue the isotopic moves as depicted in Figure 20. No matter in which of the four cases, there are an $h \in\{1, \ldots, 2 k-2 x\}$, an $x \in\{1, \ldots, k\}$, an $s \in$ $\{1, \ldots, 2 k-2 x-1\}$ and an $\varepsilon \in\{0,1\}$ such that $E^{m}(2 k, 2 k(n+f)+j)$ is equivalent to $D^{s}(2 k-2 x,(2 k-2 x)(n+f)+h) \sqcup U_{\varepsilon}$, where $U_{0}$ is a circle in the plane and $U_{1}$ is the empty set. We give $E^{m}(2 k, 2 k(n+f)+j)$ an orientation such that all crossings of $D^{S}(2 k-2 x,(2 k-2 x)(n+f)+h) \sqcup U_{\varepsilon}$ are positive. We call the diagram 

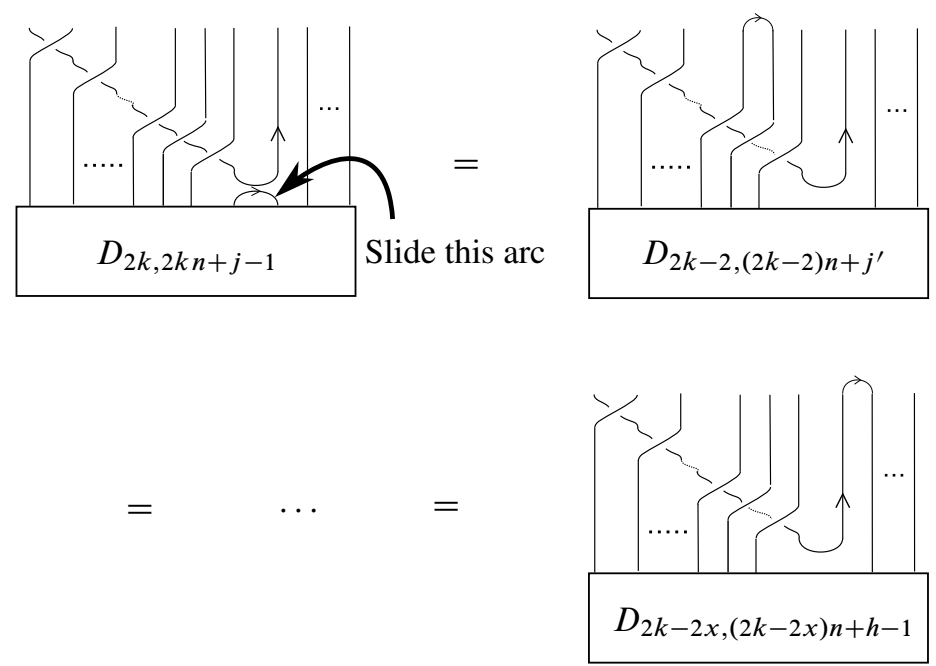

Figure 15: The diagram $E^{m}(2 k, 2 k(n+f)+j)$ can be changed to a positive diagram $D^{s}(2 k-2 x,(2 k-2 x)(n+f)+h) \sqcup U_{\varepsilon}$ (type 1$)$
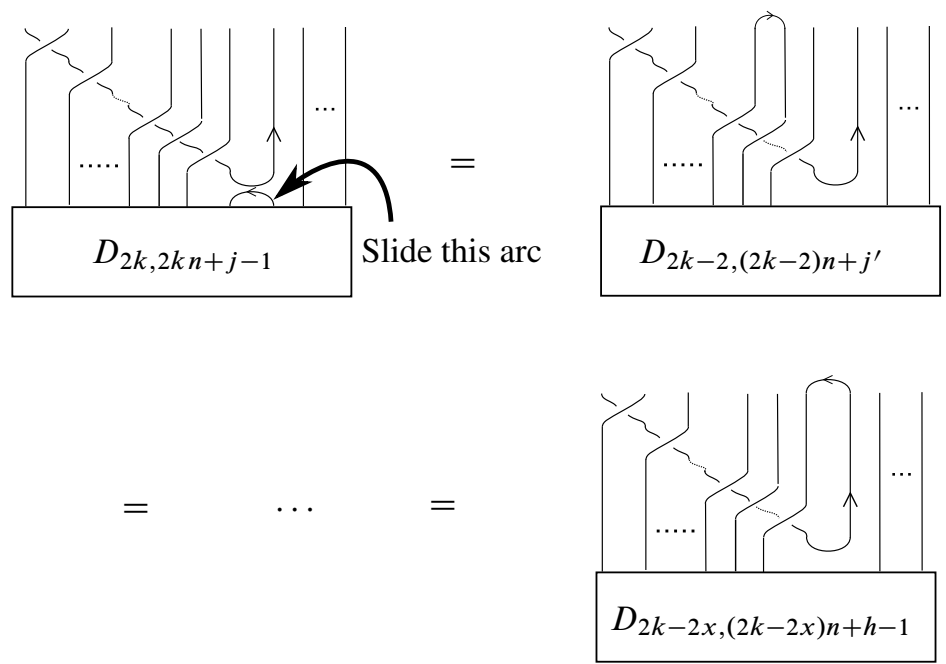

Figure 16: The diagram $E^{m}(2 k, 2 k(n+f)+j)$ can be changed to a positive diagram $D^{s}(2 k-2 x,(2 k-2 x)(n+f)+h) \sqcup U_{\varepsilon}$ (type 2$)$

$E^{m}(2 k, 2 k(n+f)+j)$ of type 1 , type 2 , type 3 and type 4 if it changes to the positive diagram as in Figures 15, 16, 19 and 20, respectively.

Now we have supposed that for $1 \leq g<k, j=1, \ldots, 2 g$ and $m=0, \ldots, 2 g-1$ we have $H^{i}\left(D^{m}(2 g, 2 g(n+f)+j)\right)=0$ if $i>2 g^{2}(n-l+1)+l(2 g)^{2}$ and $n \geq l$, or $i>l(2 g)^{2}$ 

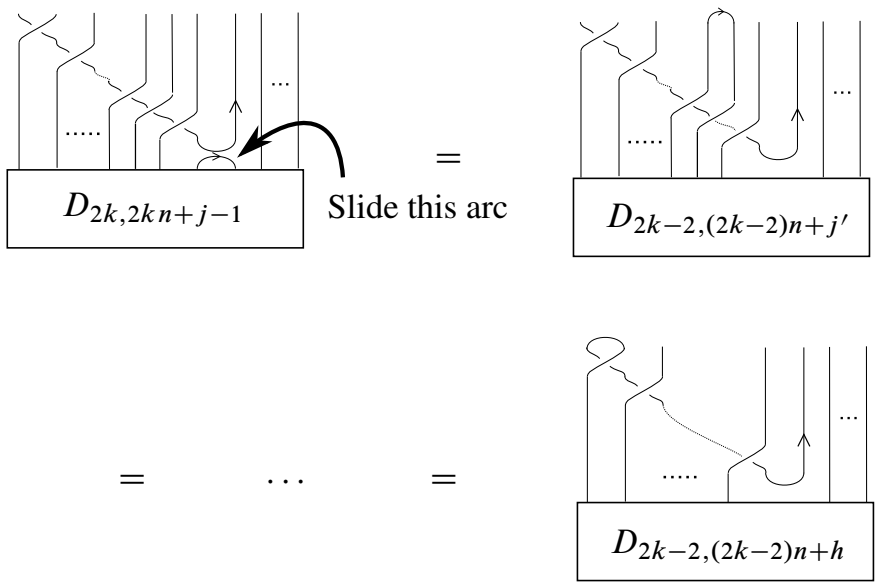

Figure 17: The diagram $E^{m}(2 k, 2 k(n+f)+j)$ can be changed to a diagram (type 3$)$
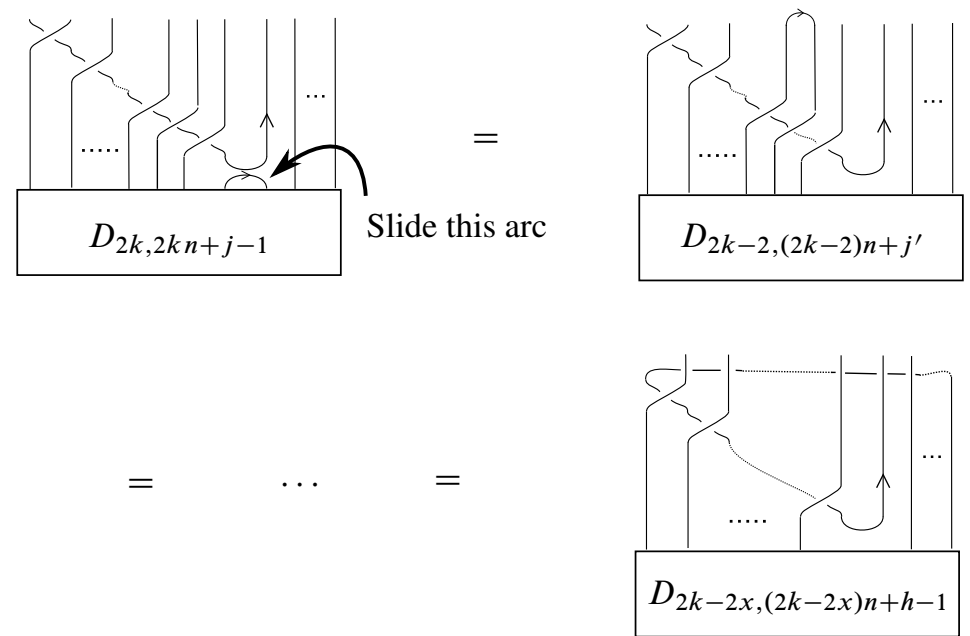

Figure 18: The diagram $E^{m}(2 k, 2 k(n+f)+j)$ can be changed to a diagram (type 4)

and $n<l$ (recall the induction hypothesis in the proof of Lemma 4.3(1)). From this induction hypothesis, if $i-n_{-}+l_{-}(2 k-2 x)^{2}>2(k-x)^{2}(n-l+1)+l(2 k-2 x)^{2}$ and $n \geq l$, or $i-n_{-}+l_{-}(2 k-2 x)^{2}>l(2 k-2 x)^{2}$ and $n<l$, then we have $H^{i}\left(E^{m}(2 k, 2 k(n+f)+j)\right)$

$$
\begin{aligned}
& =\mathrm{KH}^{i-n_{-}}\left(D^{S}(2 k-2 x,(2 k-2 x)(n+f)+h) \sqcup U_{\varepsilon}\right) \\
& =H^{i-n_{-}+l_{-}(2 k-2 x)^{2}}\left(D^{s}(2 k-2 x,(2 k-2 x)(n+f)+h) \sqcup U_{\varepsilon}\right)=0,
\end{aligned}
$$



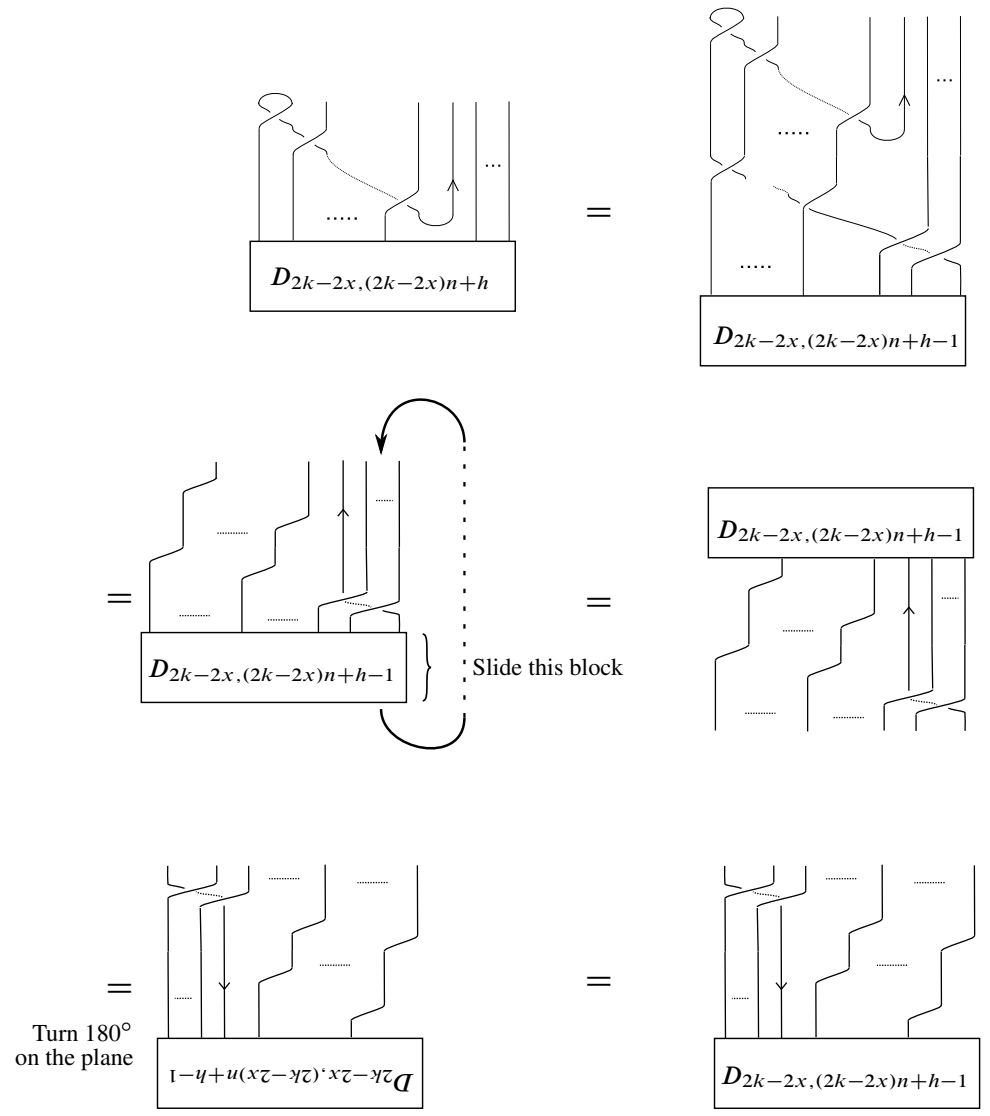

Figure 19: The diagram $E^{m}(2 k, 2 k(n+f)+j)$ can be changed to a positive diagram $D^{s}(2 k-2 x,(2 k-2 x)(n+f)+h) \sqcup U_{\varepsilon}$ (type 3$)$

where $n_{-}$is the number of the negative crossings of $E^{m}(2 k, 2 k(n+f)+j)$. Hence, to prove Claim 4.4 , it is sufficient to prove the following:

$(\mathrm{A}-1) \quad l(2 k)^{2}+2 k^{2}(n-l+1)-1$

$$
\geq 2(k-x)^{2}(n-l+1)+l_{+}(2 k-2 x)^{2}+n_{-} \quad(n \geq l),
$$

and

$$
l(2 k)^{2}-1 \geq l_{+}(2 k-2 x)^{2}+n_{-} \quad(n<l) .
$$

To prove (A-1) and (A-2), we need to count the number of the negative crossings of $E^{m}(2 k, 2 k(n+f)+j)$.

We first count its positive crossings by dividing it into four parts: part 1, part 2, part 3 and part 4 (see Figure 21). 

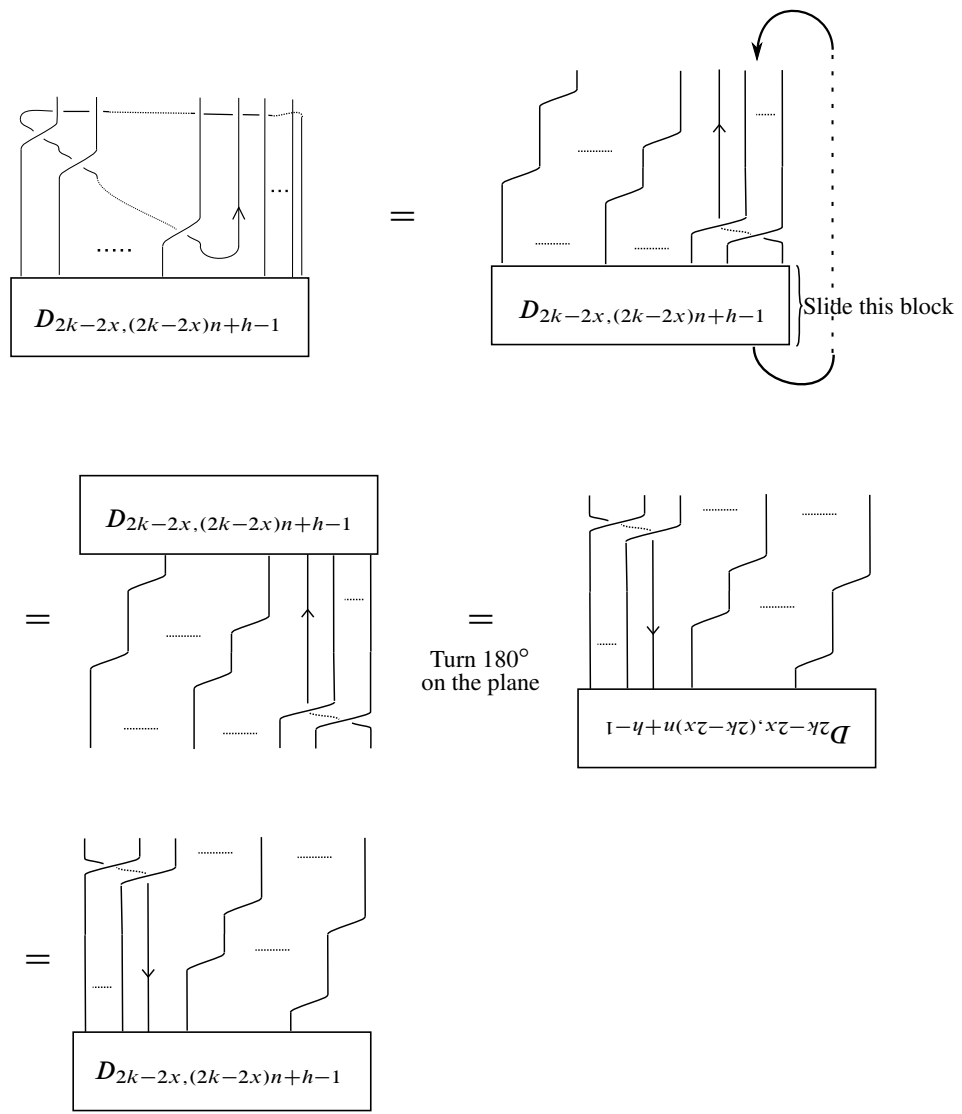

Figure 20: The diagram $E^{m}(2 k, 2 k(n+f)+j)$ can be changed to $D^{s}(2 k-$ $2 x,(2 k-2 x)(n+f)+h) \sqcup U_{\varepsilon}$ (type 4$)$

Step 1 Suppose $E^{m}(2 k, 2 k(n+f)+j)$ is either of type 1 or type 2: In part 1, we $\overline{\text { apply }} \sum_{i=0}^{x-1}(l(2 k-2 i)+l(2 k-2 i-2))$ times RII moves to $E^{m}(2 k, 2 k(n+f)+j)$ to obtain the diagram $D^{s}(2 k-2 x,(2 k-2 x)(n+f)+h) \sqcup U_{\varepsilon}$. Then the diagram $E^{m}(2 k, 2 k(n+f)+j)$ loses $\sum_{i=0}^{x-1}(l(2 k-2 i)+l(2 k-2 i-2))$ positive crossings. Moreover, $D^{s}(2 k-2 x,(2 k-2 x)(n+f)+h) \sqcup U_{\varepsilon}$ has $l_{+}(2 k-2 x)^{2}$ positive crossings in a part corresponding to part 1 . Hence, in part $1, E^{m}(2 k, 2 k(n+f)+j)$ has

$$
\sum_{i=0}^{x-1}(l(2 k-2 i)+l(2 k-2 i-2))+l_{+}(2 k-2 x)^{2}
$$

positive crossings. 


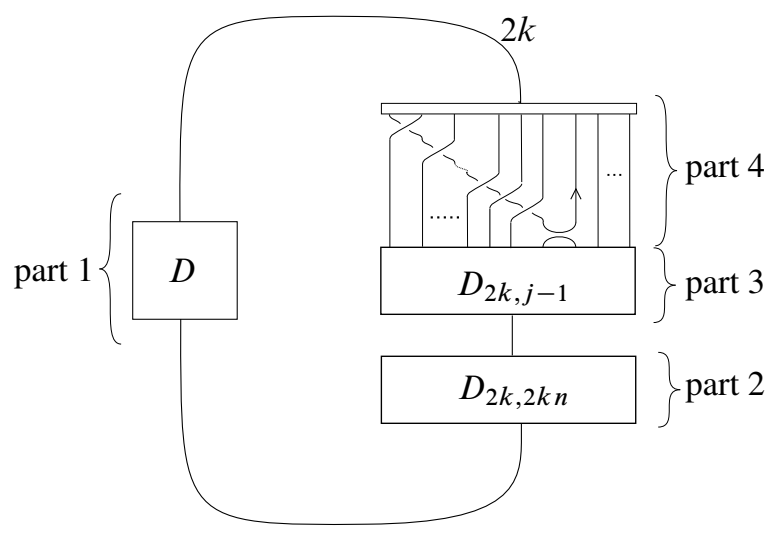

Figure 21: The diagram $E^{m}(2 k, 2 k(n+f)+j)$ divided into four parts

In part $2, E^{m}(2 k, 2 k(n+f)+j)$ has $x$ arcs directed upward and $2 k-x$ arcs directed downward (see Figure 22). Hence, in part $2, E^{m}(2 k, 2 k(n+f)+j)$ has $x(x-1) n+(2 k-x)(2 k-x-1) n$ positive crossings.

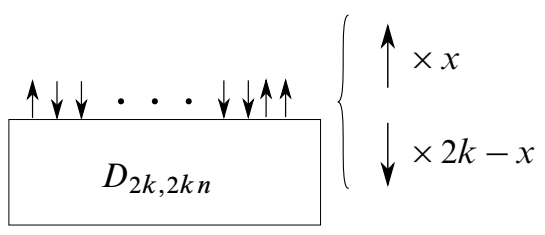

Figure 22: If $E^{m}(2 k, 2 k(n+f)+j)$ is either type 1 or type 2, in part 2, $E^{m}(2 k, 2 k(n+f)+j)$ has $x$ arcs directed upward and $2 k-x$ arcs directed downward

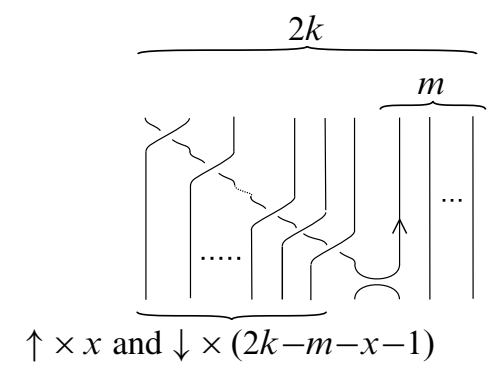

Figure 23: If $E^{m}(2 k, 2 k(n+f)+j)$ is either type 1 or type 2, in part 3, $E^{m}(2 k, 2 k(n+f)+j)$ has at least $2 k-m-1-x$ positive crossings. This figure is a minimal case.

In part $3, E^{m}(2 k, 2 k(n+f)+j)$ has at least $2 k-m-1-x$ positive crossings (see Figure 23). 
In part 4, note that there are $x$ arcs directed upward and $2 k-x$ arcs directed downward. Assume that $b$ is the number of the positions where the leftmost arc is directed upward and that $a$ is the number of the positions where the leftmost arc is directed downward (see Figure 24). Note that $a+b=j-1$ and that $b \leq x$.

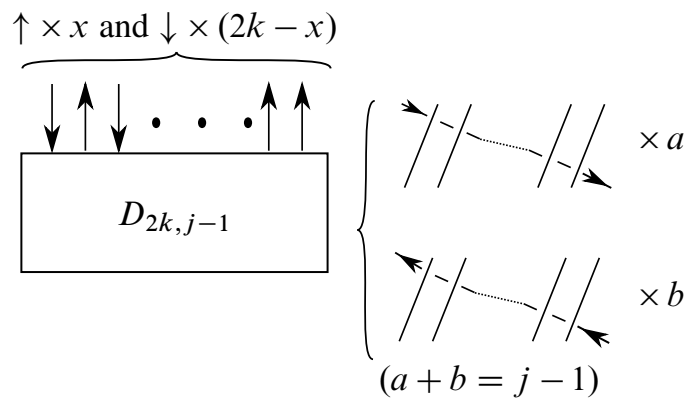

Figure 24: In the case where the diagram $E^{m}(2 k, 2 k(n+f)+j)$ is of type 1 or type 2 . In part $4, E^{m}(2 k, 2 k(n+f)+j)$ has $x$ arcs directed upward and $2 k-x$ arcs directed downward. The number of the positions where the left most arc is directed upward is $b$. The number of the positions where the left most arc is directed downward is $a$.

Then, in part $4, E^{m}(2 k, 2 k(n+f)+j)$ has

$$
b(x-1)+a(2 k-x-1)=b(x-1)+(j-1-b)(2 k-x-1)
$$

positive crossings.

Hence the diagram $E^{m}(2 k, 2 k(n+f)+j)$ has at least $X_{1}$ positive crossings, where

$$
\begin{aligned}
X_{1}=\sum_{i=0}^{x-1}(l(2 k-2 i)+l(2 k-2 i-2))+l_{+} & (2 k-2 x)^{2} \\
& +x(x-1) n+(2 k-x)(2 k-x-1) n \\
& +2 k-1-m-x \\
& +b(x-1)+(j-1-b)(2 k-x-1) .
\end{aligned}
$$

From the above discussion $E^{m}(2 k, 2 k(n+f)+j)$ has at most $X_{2}$ negative crossings, where

$$
X_{2}=l(2 k)^{2}+(2 k-1)(2 k n+j)-m-X_{1} .
$$

Then for $j \neq 2 k$ we can check the following.

$$
\begin{aligned}
l(2 k)^{2}+2 k^{2}(n-l+1)-1 & \geq 2(k-x)^{2}(n-l+1)+l_{+}(2 k-2 x)^{2}+X_{2} \quad(n \geq l), \\
l(2 k)^{2}-1 & \geq l_{+}(2 k-2 x)^{2}+X_{2} \quad(n<l) .
\end{aligned}
$$


Indeed, we can compute

$$
\begin{aligned}
l(2 k)^{2}+2 k^{2}(n-l+1)-1-\left(2(k-x)^{2}(n-l\right. & \left.+1)+l_{+}(2 k-2 x)^{2}+X_{2}\right) \\
& =2(k-x)(x-b)+x(2 k-j)-1 .
\end{aligned}
$$

We obtain $2(k-x)(x-b)+x(2 k-j)-1 \geq 0$ since $0<j<2 k, b \leq x \leq k$ and $x \geq 1$. Similarly $l_{+}(2 k-2 x)^{2}+X_{2} \leq l(2 k)^{2}-1$ for $j \neq 2 k$. This implies that (A-1) and (A-2) are true if $j \neq 2 k$ and $E^{m}(2 k, 2 k(n+f)+j)$ is either of type 1 or type 2 .

Finally we consider the case $j=2 k$. If $j=2 k$, then $x=1$ and $E^{m}(2 k, 2 k(n+f)+j)$ has $n_{-}=2(2 k-1)(n+1)-1+2 l_{+}(2 k-1)+l_{-}\left((2 k)^{2}-2(2 k-1)\right)$ negative crossings. In this case we have $l_{+}(2 k-2)^{2}+2(k-1)^{2}(n-l+1)+n_{-}=l(2 k)^{2}+2 k^{2}(n-l+1)-1$. Similarly, in this case, we obtain $l(2 k)^{2}-1 \geq l_{+}(2 k-2 x)^{2}+n_{-}$for $n<l$. These imply that (A-1) and (A-2) are true for $j=2 k$.

Step 2 Suppose $E^{m}(2 k, 2 k(n+f)+j)$ is either of type 3 or type 4: By the same

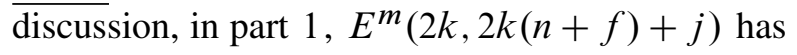

$$
\sum_{i=0}^{x-1}(l(2 k-2 i)+l(2 k-2 i-2))+l_{+}(2 k-2 x)^{2}
$$

positive crossings.

In part $2, E^{m}(2 k, 2 k(n+f)+j)$ has $2 k-x$ arcs directed upward and $x$ arcs directed downward (see Figure 25). Hence, in part $2, E^{m}(2 k, 2 k(n+f)+j)$ has $x(x-1) n+(2 k-x)(2 k-x-1) n$ positive crossings.

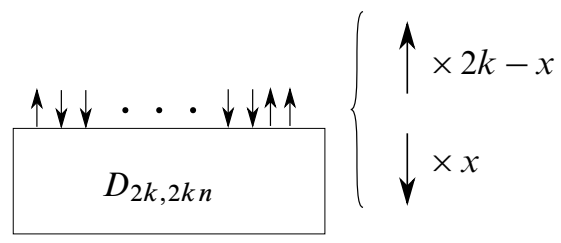

Figure 25: If $E^{m}(2 k, 2 k(n+f)+j)$ is either type 3 or type 4, in part 2, $E^{m}(2 k, 2 k(n+f)+j)$ has $2 k-x$ arcs directed upward and $x$ arcs directed downward

In part $3, E^{m}(2 k, 2 k(n+f)+j)$ may have no positive crossing.

In part 4 , note that there are $2 k-x$ arcs directed upward and $x$ arcs directed downward. Assume that $a$ is the number of the positions where the left most arc is directed upward and that $b$ is the number of the positions where the left most arc is directed downward (see Figure 26). Note that $a+b=j-1$ and that $b<x$ (we have $b \neq x$ since in part 4 the left most bottom arc is directed downward). 


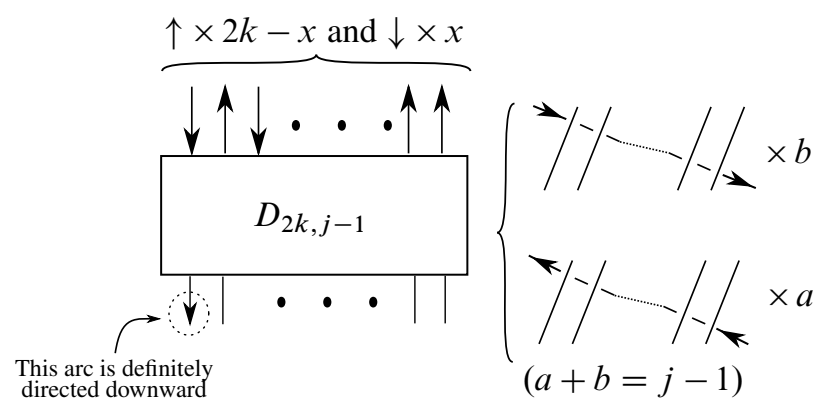

Figure 26: In the case where the diagram $E^{m}(2 k, 2 k(n+f)+j)$ is type 3 or type 4 . In part $4, E^{m}(2 k, 2 k(n+f)+j)$ has $2 k-x$ arcs directed upward and $x$ arcs directed downward. The number of the positions where the left most arc is directed upward is $a$. The number of the positions where the left most arc is directed downward is $b$. The left most bottom arc is directed downward since we give $E^{m}(2 k, 2 k(n+f)+j)$ such an orientation, (see Figures 19, 20 or 21).

Then, in part $4, E^{m}(2 k, 2 k(n+f)+j)$ has

$$
b(x-1)+a(2 k-x-1)=b(x-1)+(j-1-b)(2 k-x-1)
$$

positive crossings.

Hence the diagram $E^{m}(2 k, 2 k(n+f)+j)$ has at least $X_{1}^{\prime}$ positive crossings, where

$$
\begin{aligned}
X_{1}^{\prime}=\sum_{i=0}^{x-1}(l(2 k-2 i)+l(2 k-2 i-2))+l_{+} & (2 k-2 x)^{2} \\
& +x(x-1) n+(2 k-x)(2 k-x-1) n \\
& +b(x-1)+(j-1-b)(2 k-x-1) .
\end{aligned}
$$

From the above discussion, $E^{m}(2 k, 2 k(n+f)+j)$ has at most $X_{2}^{\prime}$ negative crossings, where

$$
X_{2}^{\prime}=l(2 k)^{2}+(2 k-1)(2 k n+j)-m-X_{1}^{\prime} .
$$

Then for $j \neq 2 k$ we can also check the following:

$$
\begin{aligned}
l(2 k)^{2}+2 k^{2}(n-l+1)-1 & \geq 2(k-x)^{2}(n-l+1)+l_{+}(2 k-2 x)^{2}+X_{2}^{\prime} \quad(n \geq l), \\
l(2 k)^{2}-1 & \geq l_{+}(2 k-2 x)^{2}+X_{2}^{\prime} \quad(n<l) .
\end{aligned}
$$

Indeed, we can compute

$$
\begin{aligned}
l(2 k)^{2}+2 k^{2}(n-l+1)-1-\left(2(k-x)^{2}(n-l+1)+l_{+}(2 k-2 x)^{2}+X_{2}^{\prime}\right) \\
=2(k-x)(x-b-1)+x(2 k-j-1)+m .
\end{aligned}
$$


We obtain $2(k-x)(x-b-1)+x(2 k-j-1)+m \geq m>0$ since we have $0<j<2 k$, $b<x \leq k$ and $x \geq 1$. Similarly $l_{+}(2 k-2 x)^{2}+X_{2}^{\prime} \leq l(2 k)^{2}-1$.

From Steps 1 and 2, we finish this proof.

Proof of Claim 4.5 The proof of Claim 4.5 is the same as that of Claim 4.4.

By the same discussion, there are an $h \in\{1, \ldots, 2 k+1-2 x\}$, an $x \in\{1, \ldots, k\}$, an $s \in\{1, \ldots, 2 k-2 x\}$ and an $\varepsilon \in\{0,1\}$ such that $E^{m}(2 k+1,(2 k+1)(n+f)+j)$ is equivalent to $D^{s}(2 k+1-2 x,(2 k+1-2 x)(n+f)+h) \sqcup U_{\varepsilon}$, where $U_{0}$ is a circle in the plane and $U_{1}$ is the empty set. We give $E^{m}(2 k+1,(2 k+1)(n+f)+j)$ an orientation such that all crossings of $D^{s}(2 k+1-2 x,(2 k+1-2 x)(n+f)+h) \sqcup U_{\varepsilon}$ are positive.

Now we have supposed that for $1 \leq g<k, j=1, \ldots, 2 g+1$ and $m=0, \ldots, 2 g$ we have $H^{i}\left(D^{m}(2 g+1,(2 g+1)(n+f)+j)\right)=0$ if $i>2 g(g+1)(n-l+1)+l(2 g+1)^{2}$ and $n \geq l$, or $i>l(2 g+1)^{2}$ and $n<l$ (recall the induction hypothesis in the proof of Lemma 4.3(2)). From this induction hypothesis, if $i-n_{-}+l_{-}(2 k+1-2 x)^{2}>$ $2(k-x)(k-x+1)(n-l+1)+l(2 k+1-2 x)^{2}$ and $n \geq l$, or $i-n_{-}+l_{-}(2 k+1-2 x)^{2}>$ $l(2 k+1-2 x)^{2}$ and $n<l$, then we have

$$
H^{i}\left(E^{m}(2 k+1,(2 k+1)(n+f)+j)\right)=0,
$$

where $n_{-}$is the number of the negative crossings of $E^{m}(2 k+1,(2 k+1)(n+f)+j)$. Hence, to prove Claim 4.5, it is sufficient to prove the following:

(A-3) $l(2 k+1)^{2}+2 k(k+1)(n-l+1)-1$

$$
\geq 2(k-x)(k+1-x)(n-l+1)+l_{+}(2 k+1-2 x)^{2}+n_{-} \quad(n \geq l),
$$

and

$$
l(2 k+1)^{2}-1 \geq l_{+}(2 k+1-2 x)^{2}+n_{-} \quad(n<l) .
$$

To prove (A-3) and (A-4), we need to count the number of the negative crossings of $E^{m}(2 k+1,(2 k+1)(n+f)+j)$.

We first count its positive crossings by dividing it into four parts as the proof of Claim 4.4.

Step 1 Suppose $E^{m}(2 k+1,(2 k+1)(n+f)+j)$ is either of type 1 or type 2 : In $\overline{\text { part } 1,} E^{m}(2 k+1,(2 k+1)(n+f)+j)$ has

$$
\sum_{i=0}^{x-1}(l(2 k+1-2 i)+l(2 k-2 i-1))+l_{+}(2 k+1-2 x)^{2}
$$


positive crossings.

In part $2, E^{m}(2 k+1,(2 k+1)(n+f)+j)$ has $x(x-1) n+(2 k+1-x)(2 k-x) n$ positive crossings.

In part $3, E^{m}(2 k+1,(2 k+1)(n+f)+j)$ has at least $2 k-m-x$ positive crossings (cf Figure 23).

In part $4, E^{m}(2 k+1,(2 k+1)(n+f)+j)$ has

$$
b(x-1)+a(2 k-x)=b(x-1)+(j-1-b)(2 k-x)
$$

positive crossings.

Hence the diagram $E^{m}(2 k+1,(2 k+1)(n+f)+j)$ has at least $Y_{1}$ positive crossings, where

$$
\begin{aligned}
Y_{1}=\sum_{i=0}^{x-1}(l(2 k+1-2 i)+l(2 k-2 i-1))+ & +(2 k+1-2 x)^{2} \\
& +x(x-1) n+(2 k+1-x)(2 k-x) n \\
& +2 k-m-x \\
& +b(x-1)+(j-1-b)(2 k-x) .
\end{aligned}
$$

From the above discussion, $E^{m}(2 k+1,(2 k+1)(n+f)+j)$ has at most $Y_{2}$ negative crossings, where

$$
Y_{2}=l(2 k+1)^{2}+2 k((2 k+1) n+j)-m-Y_{1} .
$$

Then for $j \neq 2 k+1$ we can check the following:

$l(2 k+1)^{2}+2 k(k+1)(n-l+1)-1$

$$
\geq 2(k-x)(k-x+1)(n-l+1)+l_{+}(2 k+1-2 x)^{2}+Y_{2} \quad(n \geq l),
$$

and

$$
l(2 k+1)^{2}-1 \geq l_{+}(2 k+1-2 x)^{2}+Y_{2} \quad(n<l) .
$$

Finally we consider the case where $j=2 k+1$. If $j=2 k+1$ then $x=1$ and $E^{m}(2 k+1,(2 k+1)(n+f)+j)$ has $n_{-}=4 k(n+1)-1+4 l_{+} k+l_{-}\left((2 k+1)^{2}-4 k\right)$ negative crossings. In this case we have

$$
l_{+}(2 k-1)^{2}+2 k(k-1)(n-l+1)+n_{-}=l(2 k+1)^{2}+2 k(k+1)(n-l+1)-1 .
$$

Similarly, in this case, we obtain $l(2 k+1)^{2}-1 \geq l_{+}(2 k+1-2 x)^{2}+n_{-}$for $n<l$. These imply that (A-3) and (A-4) are true for $j=2 k+1$. 
Step 2 Suppose $E^{m}(2 k+1,(2 k+1)(n+f)+j)$ is either of type 3 or type 4 :

By the same discussion, in part $1, E^{m}(2 k+1,(2 k+1)(n+f)+j)$ has

$$
\sum_{i=0}^{x-1}(l(2 k+1-2 i)+l(2 k-2 i-1))+l_{+}(2 k+1-2 x)^{2}
$$

positive crossings.

In part $2, E^{m}(2 k+1,(2 k+1)(n+f)+j)$ has $x(x-1) n+(2 k+1-x)(2 k-x) n$ positive crossings.

In part $3, E^{m}(2 k+1,(2 k+1)(n+f)+j)$ may have no positive crossing.

In part $4, E^{m}(2 k+1,(2 k+1)(n+f)+j)$ has

$$
b(x-1)+a(2 k-x)=b(x-1)+(j-1-b)(2 k-x)
$$

positive crossings.

Hence the diagram $E^{m}(2 k+1,(2 k+1)(n+f)+j)$ has at least $Y_{1}^{\prime}$ positive crossings, where

$$
\begin{aligned}
Y_{1}^{\prime}=\sum_{i=0}^{x-1}(l(2 k+1-2 i)+l(2 k-2 i-1))+ & l_{+}(2 k+1-2 x)^{2} \\
& +x(x-1) n+(2 k+1-x)(2 k-x) n \\
& +b(x-1)+(j-1-b)(2 k-x) .
\end{aligned}
$$

From the above discussion, $E^{m}(2 k+1,(2 k+1)(n+f)+j)$ has at most $Y_{2}^{\prime}$ negative crossings, where

$$
Y_{2}^{\prime}=l(2 k+1)^{2}+2 k((2 k+1) n+j)-m-Y_{1}^{\prime} .
$$

Then for $j \neq 2 k+1$ we can also check the following:

$$
\begin{aligned}
l(2 k+1)^{2}+ & 2 k(k+1)(n-l+1)-1 \\
& \geq 2(k-x)(k-x+1)(n-l+1)+l_{+}(2 k+1-2 x)^{2}+Y_{2}^{\prime} \quad(n \geq l),
\end{aligned}
$$

and

$$
l(2 k+1)^{2}-1 \geq l_{+}(2 k+1-2 x)^{2}+Y_{2}^{\prime} \quad(n<l) .
$$

From Steps 1 and 2, we finish this proof. 
Proof of Claim 4.7 In the proof of Claim 4.4, we have proved that:

- There are an $h \in\{1, \ldots, 2 k-2 x\}$, an $x \in\{1, \ldots, k\}$, an $s \in\{1, \ldots, 2 k-2 x-1\}$ and an $\varepsilon \in\{0,1\}$ such that $E^{m}(2 k, 2 k(n+f)+j)$ is equivalent to the diagram $D^{s}(2 k-2 x,(2 k-2 x)(n+f)+h) \sqcup U_{\varepsilon}$, where $U_{0}$ is a circle in the plane and $U_{1}$ is the empty set.

- If $E^{m}(2 k, 2 k(n+f)+j)$ is either of type 1 or type 2 , then it has at most $X_{2}$ negative crossings.

- If $E^{m}(2 k, 2 k(n+f)+j)$ is either of type 3 or type 4 , then it has at most $X_{2}^{\prime}$ negative crossings.

From Lemma 4.3, if $i-n_{-}+l_{-}(2 k-2 x)^{2}>2(k-x)^{2}(n-l+1)+l(2 k-2 x)^{2}$ and $n \geq l$, then we have

$$
H^{i}\left(E^{m}(2 k, 2 k(n+f)+j)\right)
$$

$$
=H^{i-n_{-}+l_{-}(2 k-2 x)^{2}}\left(D^{s}(2 k-2 x,(2 k-2 x)(n+f)+h) \sqcup U_{\varepsilon}\right)=0,
$$

where $n_{-}$is the number of the negative crossings of $E^{m}(2 k, 2 k(n+f)+j)$. In particular, if $i>2(k-x)^{2}(n-l+1)+l_{+}(2 k-2 x)^{2}+n_{-}$and $n \geq l$, then we have

$$
H^{i}\left(E^{m}(2 k, 2 k(n+f)+j)\right)=0 .
$$

From the above results, to prove Claim 4.7, it is sufficient to prove that:

(1) If $E^{m}(2 k, 2 k(n+f)+j)$ is either of type 1 or type 2 , then

$$
l(2 k)^{2}+2 k^{2}(n-l)-2 \geq 2(k-x)^{2}(n-l+1)+l_{+}(2 k-2 x)^{2}+X_{2} .
$$

(2) If $E^{m}(2 k, 2 k(n+f)+j)$ is either of type 3 or type 4 , then

$$
l(2 k)^{2}+2 k^{2}(n-l)-2 \geq 2(k-x)^{2}(n-l+1)+l_{+}(2 k-2 x)^{2}+X_{2}^{\prime} .
$$

We have already proved (2) in the proof of Claim 4.4. Let us prove (1). Recall $j=1, \ldots, 2 k-1, b \leq x \leq k$ and $x \geq 1$. Hence, if $j \leq 2 k-2$ or $x \geq 2$, we obtain

$$
\begin{aligned}
l(2 k)^{2}+2 k^{2}(n-l)-2-\left(2(k-x)^{2}(n-l+1)+l_{+}(2 k-2 x)^{2}+X_{2}\right) & \\
& =-2+x(2 k-j)+2(k-x)(b-x) \geq 0 .
\end{aligned}
$$

If $j=2 k-1$ and $x=1$, then $E^{m}(2 k, 2 k(n+f)+j)$ is either of type 3 or type 4 . Hence we obtain $l(2 k)^{2}+2 k^{2}(n-l)-2-\left(2(k-x)^{2}(n-l+1)+l_{+}(2 k-2 x)^{2}+X_{2}\right) \geq 0$ if $E^{m}(2 k, 2 k(n+f)+j)$ is either of type 1 or type 2 . 
Proof of Lemma 3.10 To prove Lemma 3.10, we use Lemma A.1 below. It follows from Lemma A.1 that

$$
H^{2 k(k+1) n}\left(D_{2 k+1,(2 k+1) n-1}\right)=H^{2 k(k+1) n}\left(D_{2 k+1,(2 k+1)(n-1)}\right)
$$

for any positive integers $n$ and $k$. From Lemma 4.2, the right-hand side is zero.

Lemma A.1 Let $K$ be a knot and $D$ be a knot diagram with $l_{+}$positive crossings and $l_{-}$negative crossings. Put $l=l_{+}+l_{-}$and $f=l_{+}-l_{-}$. Then for any positive integer $k$ and any $n>l$, we obtain

$$
\begin{aligned}
H^{2 k(k+1)(n+l)+l}(D(2 k+1, & (2 k+1)(n+f)-1)) \\
= & H^{2 k(k+1)(n+l)+l}(D(2 k+1,(2 k+1)(n+f-1))) .
\end{aligned}
$$

Proof We first compute $H^{i}\left(E^{m}(2 k+1,(2 k+1)(n+f-1)+j)\right)$. In the proof of Claim 4.5, we have proved that:

- There are an $h \in\{1, \ldots, 2 k+1-2 x\}$, an $x \in\{1, \ldots, k\}$, an $s \in\{1, \ldots, 2 k-2 x\}$ and an $\varepsilon \in\{0,1\}$ such that $E^{m}(2 k+1,(2 k+1)(n+f)+j)$ is equivalent to $D^{s}(2 k+1-2 x,(2 k+1-2 x)(n+f)+h) \sqcup U_{\varepsilon}$, where $U_{0}$ is a circle in the plane and $U_{1}$ is the empty set.

- If $E^{m}(2 k+1,(2 k+1)(n+f)+j)$ is either of type 1 or type 2 , then it has at most $Y_{2}$ negative crossings.

- If $E^{m}(2 k+1,(2 k+1)(n+f)+j)$ is either of type 3 or type 4 , then it has at most $Y_{2}^{\prime}$ negative crossings.

From Lemma 4.3, if

$$
i-n_{-}+l_{-}(2 k+1-2 x)^{2}>2(k-x)(k-x+1)(n-l+1)+l(2 k+1-2 x)^{2}
$$

and $n \geq l$, then we have

$$
\begin{aligned}
& H^{i}\left(E^{m}(2 k+1,(2 k+1)(n+f)+j)\right) \\
& \quad=H^{i-n_{-}+l_{-}(2 k+1-2 x)^{2}}\left(D^{s}(2 k+1-2 x,(2 k+1-2 x)(n+f)+h)\right)=0,
\end{aligned}
$$

where $n_{-}$is the number of the negative crossings of $E^{m}(2 k+1,(2 k+1)(n+f)+j)$. In particular, if $i>2(k-x)(k-x+1)(n-l+1)+l_{+}(2 k+1-2 x)^{2}+n_{-}$and $n \geq l$, then we have

$$
H^{i}\left(E^{m}(2 k+1,(2 k+1)(n+f)+j)\right)=0 .
$$

Then we can prove the following claim. 
Claim A.2 For $j=1, \ldots, 2 k$ and $m=1, \ldots, 2 k$, if $E^{m}(2 k+1,(2 k+1)(n+f)+j)$ is either of type 1 or type 2 , then

(A-5) $l(2 k+1)^{2}+2 k(k+1)(n-l)-2$

$$
\begin{aligned}
& \geq 2(k-x)(k-x+1)(n-l+1)+l_{+}(2 k+1-2 x)^{2}+Y_{2} \\
& \geq 2(k-x)(k-x+1)(n-l+1)+l_{+}(2 k+1-2 x)^{2}+n_{-},
\end{aligned}
$$

and if $E^{m}(2 k+1,(2 k+1)(n+f)+j)$ is either of type 3 or type 4 , then (A-6) $l(2 k+1)^{2}+2 k(k+1)(n-l)-2$

$$
\begin{aligned}
& \geq 2(k-x)(k-x+1)(n-l+1)+l_{+}(2 k+1-2 x)^{2}+Y_{2}^{\prime} \\
& \geq 2(k-x)(k-x+1)(n-l+1)+l_{+}(2 k+1-2 x)^{2}+n_{-} .
\end{aligned}
$$

We prove Claim A.2 later. From the above discussion and Claim A.2, if

$$
i>l(2 k+1)^{2}+2 k(k+1)(n-l)-2,
$$

then $H^{i}\left(E^{m}(2 k+1,(2 k+1)(n+f)+j)\right)=0$ for $j=1, \ldots, 2 k$ and $m=1, \ldots, 2 k$. Now there is the following exact sequence:

$$
\begin{aligned}
\cdots & \longrightarrow H^{i-1}\left(E^{m}(2 k+1,(2 k+1)(n+f-1)+j)\right) \\
& \longrightarrow H^{i}\left(D^{m-1}(2 k+1,(2 k+1)(n+f-1)+j)\right) \\
& \longrightarrow H^{i}\left(D^{m}(2 k+1,(2 k+1)(n+f-1)+j)\right) \\
& \longrightarrow H^{i}\left(E^{m}(2 k+1,(2 k+1)(n+f-1)+j)\right) \longrightarrow \cdots,
\end{aligned}
$$

where $m=1, \ldots, 2 k, n \geq 0$ and $j=1, \ldots, 2 k$. From the above result and this exact sequence, we obtain

$$
\begin{aligned}
H^{2 k(k+1)(n+l)+l}( & D(2 k+1,(2 k+1)(n+f)-1)) \\
& =H^{2 k(k+1)(n+l)+l}\left(D^{1}(2 k+1,(2 k+1)(n+f-1)+2 k-1)\right) \\
& =\cdots \\
& =H^{2 k(k+1)(n+l)+l}\left(D^{2 k}((2 k+1,(2 k+1)(n+f-1)+2 k-1))\right. \\
& =H^{2 k(k+1)(n+l)+l}\left(D^{0}(2 k+1,(2 k+1)(n+f-1)+2 k-2)\right) \\
& =\cdots \\
& =H^{2 k(k+1)(n+l)+l}(D(2 k, 2 k(n+f-1))) .
\end{aligned}
$$

Proof of Claim A.2 We have already proved (A-6) in the proof of Claim 4.5. Let us prove (A-5). Recall $j=1, \ldots, 2 k+1, b \leq x \leq k$ and $x \geq 1$. Hence if $j \leq 2 k-1$ 
or $x \geq 2$, we obtain

$$
\begin{array}{r}
l(2 k+1)^{2}+2 k(k+1)(n-l)-2-\left(2(k-x)(k-x+1)(n-l+1)+l_{+}(2 k+1-2 x)^{2}+Y_{2}\right) \\
=-2+x(2 k+1-j)+2(k-x)(b-x)+x-b \geq 0 .
\end{array}
$$

If $j=2 k$ and $x=1$, then $E^{m}(2 k+1,(2 k+1)(n+f)+j)$ is either of type 3 or type 4 . Hence if $E^{m}(2 k+1,(2 k+1)(n+f)+j)$ is either of type 1 or type 2, we obtain $l(2 k+1)^{2}+2 k(k+1)(n-l)-2 \geq 2(k-x)(k-x+1)(n-l+1)+l_{+}(2 k+1-2 x)^{2}+Y_{2}$ for $j=1, \ldots, 2 k$.

Acknowledgements The author would like to express his gratitude to Hitoshi Murakami for his encouragement. He also would like to thank Yuanyuan Bao for her helpful comments. Kálmán Tamás gave him constructive comments and warm encouragement. The author is also grateful to the referee for his/her comments. This paper is the author's master thesis at Tokyo Institute of Technology in February 2012.

\section{References}

[1] T Abe, An estimation of the alternation number of a torus knot, J. Knot Theory Ramifications 18 (2009) 363-379 MR2514849

[2] C C Adams, J F Brock, J Bugbee, T D Comar, KA Faigin, A M Huston, Almost alternating links, Topology Appl. 46 (1992) 151-165 MR1184114

[3] D Bar-Natan, On Khovanov's categorification of the Jones polynomial, Algebr. Geom. Topol. 2 (2002) 337-370 MR1917056

[4] A Champanerkar, I Kofman, Spanning trees and Khovanov homology, Proc. Amer. Math. Soc. 137 (2009) 2157-2167 MR2480298

[5] M Hedden, Knot Floer homology of Whitehead doubles, Geom. Topol. 11 (2007) 2277-2338 MR2372849

[6] M Khovanov, A categorification of the Jones polynomial, Duke Math. J. 101 (2000) 359-426 MR1740682

[7] M Khovanov, Patterns in knot cohomology, I, Experiment. Math. 12 (2003) 365-374 MR2034399

[8] R Kirby, P Melvin, The 3-manifold invariants of Witten and Reshetikhin-Turaev for sl(2, C), Invent. Math. 105 (1991) 473-545 MR1117149

[9] E S Lee, An endomorphism of the Khovanov invariant, Adv. Math. 197 (2005) 554-586 MR2173845

[10] W B R Lickorish, An introduction to knot theory, Graduate Texts in Mathematics 175, Springer, New York (1997) MR1472978 
[11] C Livingston, Computations of the Ozsváth-Szabó knot concordance invariant, Geom. Topol. 8 (2004) 735-742 MR2057779

[12] C Livingston, S Naik, Ozsváth-Szabó and Rasmussen invariants of doubled knots, Algebr. Geom. Topol. 6 (2006) 651-657 MR2240910

[13] J Rasmussen, Khovanov homology and the slice genus, Invent. Math. 182 (2010) 419-447 MR2729272

[14] M Stošić, Homological thickness and stability of torus knots, Algebr. Geom. Topol. 7 (2007) 261-284 MR2308944

[15] M Stošić, Khovanov homology of torus links, Topology Appl. 156 (2009) 533-541 MR2492301

[16] P Turner, A spectral sequence for Khovanov homology with an application to $(3, q)-$ torus links, Algebr. Geom. Topol. 8 (2008) 869-884 MR2443099

[17] O Viro, Remarks on definition of Khovanov homology arXiv:math/0202199

[18] O Viro, Khovanov homology, its definitions and ramifications, Fund. Math. 184 (2004) 317-342 MR2128056

Department of Mathematics, Tokyo Institute of Technology

Ookayama, Meguro, Tokyo 152-8551, Japan

tagami.k.aa@m.titech.ac.jp

Received: 23 October $2012 \quad$ Revised: 16 March 2013 